\title{
Unveiling the Impact of Nanoparticle Size Dispersity on the Behavior of Polymer Nanocomposites
}

DOI:

10.1016/j.polymer.2017.01.081

\section{Document Version}

Accepted author manuscript

Link to publication record in Manchester Research Explorer

\section{Citation for published version (APA):}

Burgos-Mármol, J. J., \& Patti, A. (2017). Unveiling the Impact of Nanoparticle Size Dispersity on the Behavior of Polymer Nanocomposites. Polymer, 113, 92-104. https://doi.org/10.1016/j.polymer.2017.01.081

\section{Published in:}

Polymer

\section{Citing this paper}

Please note that where the full-text provided on Manchester Research Explorer is the Author Accepted Manuscript or Proof version this may differ from the final Published version. If citing, it is advised that you check and use the publisher's definitive version.

\section{General rights}

Copyright and moral rights for the publications made accessible in the Research Explorer are retained by the authors and/or other copyright owners and it is a condition of accessing publications that users recognise and abide by the legal requirements associated with these rights.

\section{Takedown policy}

If you believe that this document breaches copyright please refer to the University of Manchester's Takedown Procedures [http://man.ac.uk/04Y6Bo] or contact uml.scholarlycommunications@manchester.ac.uk providing relevant details, so we can investigate your claim.

\section{OPEN ACCESS}




\title{
Unveiling the Impact of Nanoparticle Size Dispersity on the Behavior of Polymer Nanocomposites
}

\author{
J. Javier Burgos-Mármol and Alessandro Patti* \\ School of Chemical Engineering and Analytical Science, \\ The University of Manchester. Sackville Street, M13 9PL, Manchester, UK
}

\begin{abstract}
Polymer nanocomposites (PNCs), a class of polymer materials incorporating nano-sized particles (NPs), have tremendous potential in industrial formulations and technological applications, such as protective coatings and food packaging. In this work, we perform Molecular Dynamics simulations to unveil the impact of NP size dispersity on a variety of properties that characterize the response of PNCs at the nano and macro scales. In particular, at the nano scale, we investigate the space distribution of small and large polydisperse NPs and their ability to diffuse through a dense isotropic distribution of unentangled polymer chains. We find very interesting scaling laws relating the average size and polydispersity index of NPs with their diffusion coefficients, generally underestimated by existing theoretical models. These theories are here adapted to include the effect of NP size dispersity and their predictions, confirming the relevance of incorporating such contributions, are validated against our simulation results. We also analyze the diffusivity of the polymer chains as a function of the interparticle distance for a spectrum of NP diameters and confirmed the existence of a single master curve as recently observed experimentally (S. Gam et al., Soft Matter, 2012, 8, 6512). To assess the effect of NP size dispersity on the macroscopic response of our model PNC, we evaluate two key transport properties, shear viscosity and thermal conductivity, which are found to display an intriguing universal behavior when plotted against the polymer/NP specific interface area and the inverse of the NP's mass, respectively.
\end{abstract}

Keywords: Polymer Nanocomposites, Nanoparticles, Molecular Dynamics, Diffusion, Viscosity, Thermal Conductivity

\section{INTRODUCTION}

Polymer nanocomposites (PNCs) are materials composed of a polymer matrix incorporating particles with at least one dimension in the nanometer scale [1]. The presence of such nanofillers can dramatically alter the local dynamics and morphology of the polymer chains as well as the macroscopic response of the polymer, including its mechanical, thermal, and rheological properties. If rationally formulated, PNCs can have a high impact on a number of key industrial applications, including, but not limited to, coatings, paints, electronics, food packaging, and personal care [2-11]. The origin of such remarkable properties as compared to a pristine polymer is usually ascribed to the delicate balance between enthalpic and entropic forces established at the nanoscale between host and guest. More specifically, the small size of the nanoparticles (NPs) provides larger surface to volume ratios as compared to traditional composites. As a consequence, the interface between NPs and polymer increases and a larger number of chains can interact with the NPs. Additionally, the $\mathrm{NP} /$ polymer relative size controls the relaxation of the polymer chains across several time scales and influences their response to external stimuli. Equally noticeable is the possibility to improve the performance of a polymer by adding only a little amount of NPs (generally around $5 \mathrm{wt}$. \%), so that the overall weight of the material, a crucial parameter for many industrial applications, and its cost do not increase significantly. This twofold positive effect, enhancing the material properties by keeping its weight to satisfactory levels, cannot be achieved by employing conventional microfillers.

Predicting and controlling the macroscopic behavior of PNCs relies on the full understanding of the physical phenomena taking place at the nanoscale and especially related to the mobility of chains and NPs. Generally speaking, these phenomena depend on properties intrinsic to the polymer (e.g. chain stiffness, radius of gyration and degree of entanglement), properties intrinsic to the NPs (e.g. size, shape anisotropy, polydispersity and surface modifications), and properties resulting from the combination of both species (e.g. NPs' distribution, extension of the NP/polymer interface area and interaction strength). It is perhaps the latter contribution that introduces a substantial additional complexity to the theoretical description of these systems. As a matter of fact, theoretical models that have been successfully applied to investigate the diffusion of colloidal particles in a suspension and their effect on measurable properties, such as viscosity and thermal conductivity, cannot be straightaway applied to polymer melts incorporating NPs, where a number of complex phenomena take place.

As far as diffusion is concerned, experimental [12-14] and computational [15-17] studies have reported a relevant increase in the diffusivity of neutral spherical NPs than that predicted by the Stokes-Einstein relation at NP's diameter smaller than the polymer radius of gyration, $R_{g}$. By taking into account the polymer-particle size ratio, Ganesan et al. explained this deviation by introducing a phenomenological, constitutive equation for the diffusivity of NPs in unentangled polymer melts [18]. Their model is based on the assumption, originally suggested by Wyart and de Gennes [19], that the dynamics of particles with radius $R<R_{g}$ should be described by considering the response of the polymer melt for velocity gradient scales smaller than $R_{g}$. Similarly, Yamamoto and Schweizer proposed the same dependence with different coefficients, affirming that this deviation is a result of non-hydrodynamic contributions becoming dominant for particle's diameter smaller than $R_{g}$ [20]. Liu et al. used an 
alternative definition of the hydrodynamic radius previously proposed by Ould-Kaddour and Levesque for NPs in simple liquids $[15,17]$. By implementing it into the Stokes-Einstein relation, they studied the diffusion of a coarse-grained model of NPs in a polymer melt, and obtained values that underestimated the experimental data for particle's size much smaller than $R_{g}$.

It is generally accepted that the viscosity of a solvent increases when non-interacting colloidal particles are incorporated. The predictions introduced by Einstein and subsequent corrections to his model were obtained from hydrodynamic considerations, with the solvent being treated as a continuous medium [21-27]. According to these theoretical models, a change in solvent viscosity would only depend on the particle's volume fraction, $\phi$, and would be independent of other factors, such as particle size or mass. Nevertheless, several studies show that this assumption is no longer valid at particle sizes comparable to the characteristic lengths of the polymer. Mackay et al. experimentally measured the viscosity of linear polysterene incorporating cross-linked polysterene NPs with diameter approximately equal to or smaller than the radius of gyration of the polymer chains [28]. They determined a decrease in viscosity for several values of the volume fraction of NPs. By performing molecular dynamics (MD) simulations, Kumar et al. found that it is possible to increase or decrease the viscosity of a PNC by tuning the interactions established between NPs and polymer chains [29]. These results were confirmed more recently by Patti, whose MD simulations showed a crucial dependence of the shear viscosity on the polymer-NP relative size [30]. Furthermore, Smith et al. found that the viscosity of unentangled polymers, normalized by a second degree polynomial of $\phi$, collapsed into a single master curve that strongly depended on the $\mathrm{NP} /$ polymer interactions, rather than on $\phi$ or $R_{g}$ [31]. Under these circumstances, the size dispersity of particles is expected to play an important role, especially when particles' average size and polymer structural dimensions are comparable [32].

Thermal conductivity denotes the ability of a material to transfer heat. More specifically, the larger the thermal conductivity of a material, the faster the heat transfer across it. Most of the polymers exhibit a relatively poor thermal conductivity, which is in the order of $10^{-1} \mathrm{Wm}^{-1} \mathrm{~K}^{-1}$ at ambient temperature. Adding thermally conducting NPs can enhance the thermal conductivity of polymers and extend their use to applications where dissipating heat effectively, while keeping ease of processing, flexibility of design, and low weight, is crucial [33]. Nevertheless, achieving thermal conductivities above $3 \mathrm{Wm}^{-1} \mathrm{~K}^{-1}$ is currently feasible, but significantly challenging [34]. Analogously to viscosity, thermal conductivity of polymers containing a small amount of micrometric fillers can be expressed as a function of the particle's volume fraction [35]. However, additional parameters, such as internal structure of particles and phonon scattering at the interface, are crucial to explain heat transfer across a material exposed to a temperature gradient. Theoretical models describing the thermal conductivity of a solvent containing micrometric particles usually combine the bulk thermal conductivities of both solvent and filler [36]. For instance, the parallel model estimates the thermal conductivity of the composite as the arithmetic mean of those of host and guest species [37]. Other authors included the thermal resistance at the interface of spherical and spheroidal fillers, and improved the agreement of the theory with experimental observations [38-40]. These models, however, are based on Fourier's Law, which is not valid when the mean free path of the energy carriers is larger than the size of the filler, and are only applicable in the dilute limit, where the $\mathrm{NP} / \mathrm{NP}$ interactions can be neglected. The former limitation was addressed by modifying the bulk mean free path of the energy carriers to include their multiple interface scattering with the particles [41, 42]. The second limitation was addressed few years later by explicitly incorporating the dependence of the thermal conductivity on $\phi$ into the theory formerly proposed by Bruggeman [43], and validated against experiments on epoxy resins containing silica NPs [44]. In addition, experiments by Shima and Philip showed that, very small iron oxide and silver NPs (average size $\sim 7 \mathrm{~nm}$ ) contributed equally to increase the thermal conductivity of dilute hexadecane or kerosene nanofluids $(\phi \leq 8 \%)$, regardless of the specific thermal properties of the NPs studied [45].

In this study, we address the impact of NP size dispersity on the above mentioned properties. Size dispersity is of fundamental importance as it is able, for instance, to delay or prevent crystallization of colloidal suspensions [46, 47], or improve the selectivity and specificity of drug delivery [48]. As far as PNCs are concerned, it is known that polydispersity determines the interparticle distance (ID) and, consequently, the mobility of the polymer chains when $R_{g} \simeq I D$ [49]. In particular, we investigate the effect of incorporating spherical NPs into a dense polymer matrix and unveil the impact of their polydispersity on the structure, dynamics, viscosity, and thermal conductivity of the resulting PNC. To this end, we perform molecular dynamics (MD) simulations of a coarsegrained (CG) model of polymer melts containing polydisperse spherical NPs, and show that size dispersity, which, to the best of our knowledge, has been generally neglected by the available existing theories on particles' mobility in a fluid, is crucial to estimate the diffusivity of nano-sized particles. Therefore, we provide a theoretical framework that incorporates the effect of NP's polydispersity and validate it against our simulation results. Furthermore, our study of the rheological response of the PNCs provides an insight into the effects of NP size dispersity, which resulted in a master curve describing the viscosity as a function of the specific interface area. Finally, our simulation results reveal that the mass of the NPs, which behave as mere insulating structural heterogeneities, determines the thermal response of a PNC and the universal behavior of its thermal conductivity. 


\section{MODEL}

In this work, we present a CG model of a PNC containing $N_{c}$ fully flexible linear polymer chains, and $N_{n}$ spherical NPs. Polymer chains are built up by $L_{c}=10$ identical beads of diameter $\sigma_{m}$, which is the system's unit length, and mass $M_{m}$, the system's unit mass. All NPs have a spherical shape, but different diameters $\sigma_{n_{i}}$, with $1 \leqslant i \leqslant N_{n}$, which are generated following a Log-normal (LN) probability density function (pdf) $[50,51]$. This LN pdf has been modified by introducing a cutoff diameter, $\sigma_{c}$, which avoids the presence of NPs smaller than $\sigma_{m}$, and reads

$$
f_{L N c}\left(\sigma_{n} ; \mu, \zeta, \sigma_{c}\right)=\frac{H\left(\sigma_{n}-\sigma_{c}\right)}{\Omega} f_{L N}\left(\sigma_{n} ; \mu, \zeta\right)
$$

with

$$
f_{L N}\left(\sigma_{n} ; \mu, \zeta\right)=\frac{1}{\zeta \sqrt{2 \pi}} \frac{1}{\sigma_{n}} e^{-\left(\ln \sigma_{n}-\mu\right)^{2} / 2 \zeta^{2}}
$$

where $\sigma_{n}$ is the NP's diameter, $H$ the Heaviside step function, $\sigma_{c} / \sigma_{m}=1$ the cutoff diameter, $\mu$ and $\zeta$ the mean and standard deviation, respectively, of the natural logarithm of $\sigma_{n}$, and $\Omega$ the following normalization constant:

$$
\Omega=\int_{0}^{\infty} \mathrm{d} \sigma_{n} H\left(\sigma_{n}-\sigma_{c}\right) f_{L N}\left(\sigma_{n} ; \mu, \zeta\right)
$$

We also note that the mass of each NP, $M_{n_{i}}$, scales with its volume, and $M_{n_{i}} / M_{m}=1 \Leftrightarrow \sigma_{n_{i}} / \sigma_{m}=1$. The parameters defining our NP size distributions are the average diameter, $\left\langle\sigma_{n}\right\rangle$, and the polydispersity index, $\Pi_{Ð}[52,53]$, as obtained from Eq. (2):

$$
\left\langle\sigma_{n}\right\rangle=e^{\mu+\frac{\zeta^{2}}{2}}
$$

$$
\Pi_{Ð} \equiv \frac{v}{\left\langle\sigma_{n}\right\rangle^{2}}=e^{\zeta^{2}}-1
$$

where $v=\left[\exp \left(\zeta^{2}\right)-1\right]\left[\exp \left(2 \mu+\zeta^{2}\right)\right]$ is the variance of the $\mathrm{LN}$ pdf. We note that the polydispersity index could also be defined as $\sqrt{v} /\left\langle\sigma_{n}\right\rangle$, with $\sqrt{v}$ the standard deviation of the LN pdf. However, for practical purposes, we prefer to apply Eq. (5) instead. Finally, the LN moments, which are needed to compute NP surface $\left(\left\langle\sigma_{n}^{2}\right\rangle\right)$, volume $\left(\left\langle\sigma_{n}^{3}\right\rangle\right)$ and diffusion $\left(\left\langle\sigma_{n}^{-1}\right\rangle\right)$ averages read

$$
\left\langle\sigma_{n}^{N}\right\rangle=\left\langle\sigma_{n}\right\rangle^{N}\left(1+\Pi_{Ð}\right)^{\left(N^{2}-N\right) / 2}, \forall N \in \mathbb{R}
$$

or, alternatively, including the cutoff diameter:

$$
\left\langle\sigma_{n}^{N}\right\rangle_{\sigma_{c}}=\frac{\left\langle\sigma_{n}\right\rangle^{N}}{2 \Omega} \operatorname{erfc}\left[\frac{\ln \left(\sigma_{c}\left(1+\Pi_{Ð}\right)^{1 / 2-N} /\left\langle\sigma_{n}\right\rangle\right)}{\sqrt{2 \ln \left(1+\Pi_{Ð}\right)}}\right], \forall N \in \mathbb{R}
$$

More information about the use of these pdfs can be found in the Supporting Information.

Several systems were studied for $1 \leqslant\left\langle\sigma_{n}\right\rangle / \sigma_{m} \leqslant 10$ and $0 \leqslant \Pi_{Ð} \leqslant 0.2$. The NP's volume fraction was $\phi_{n}=0.05$, with $\phi_{n}=\pi N_{n}\left\langle\sigma_{n}\right\rangle^{3}\left(1+\Pi_{Ð}\right)^{3} / 6 V, V=L^{3}$ the volume of the cubic simulation box of side $L$. The number of NPs was set at 30 to generate a smooth LN histogram for polydisperse systems, and between $N_{n}=5$ and 500 for monodisperse systems. The number density of polymer was set to $\rho_{m} / \sigma_{m}^{-3}=0.7$, with $\rho_{m}=N_{c} L_{c} / V\left(1-\phi_{n}\right)$. The force field describing the interactions established in our system is that employed by Liu et al. [17], which combines the polymer model proposed by Kremer and Grest [54] with that on NPs by Smith [55]. In particular, non-bonded interactions are treated via a modified LennardJones (LJ) potential that reads

$$
U^{\alpha \beta}(r)=\left\{\begin{array}{ll}
4 \epsilon\left[\left(\frac{\sigma_{m}}{r-R^{\alpha \beta}}\right)^{12}-\left(\frac{\sigma_{m}}{r-R^{\alpha \beta}}\right)^{6}\right]+U_{0}^{\alpha \beta}\left(R^{\alpha \beta}+r_{c}^{\alpha \beta}\right) & \text { if } r<R^{\alpha \beta}+r_{c}^{\alpha \beta} \\
0 & \text { if } r \geq R^{\alpha \beta}+r_{c}^{\alpha \beta}
\end{array},\right.
$$

where $r$ is the distance between the center of mass of two generic beads $\alpha$ and $\beta, \epsilon$ the system's energy unit, $R^{\alpha \beta}$ the closest possible distance between $\alpha$ and $\beta, r_{c}^{\alpha \beta}$ their cutoff distance, and $U_{0}^{\alpha \beta}\left(R^{\alpha \beta}+r_{c}^{\alpha \beta}\right)$ cancels the interaction at the cutoff. In particular, $R^{\alpha \beta}$ assumes different values according to the interacting pair considered, being $R^{m m}=0, R^{m n_{i}}=\left(\sigma_{n_{i}}-\sigma_{m}\right) / 2$, and $R^{n_{i} n_{j}}=\left(\sigma_{n_{i}}+\sigma_{n_{j}}\right) / 2-\sigma_{m}$. Essentially, NPs are represented by a hard core and a soft shell of thickness equal to $\xi_{n}=\sigma_{m} / 2$, as schematically depicted in Fig. 1.

The cutoff distances are set in such a way to achieve a satisfactory NPs' dispersion in the polymer melt and read $r_{c}^{m m} / \sigma_{m}=2^{1 / 6}, r_{c}^{m n_{i}} / \sigma_{m}=2.5$, and $r_{c}^{n_{i} n_{j}} / \sigma_{m}=2^{1 / 6}$. As a consequence, NPs and polymer chains establish favorable, attractive interactions, whereas NP/NP and non-bonded monomermonomer interactions are purely repulsive. Finally, in order to describe the bonded interactions between adjacent beads of polymer chains, the above LJ potential, $U^{m m}(r)$, is employed along with a FENE (Finitely Extensible Nonlinear Elastic) potential, which reads 


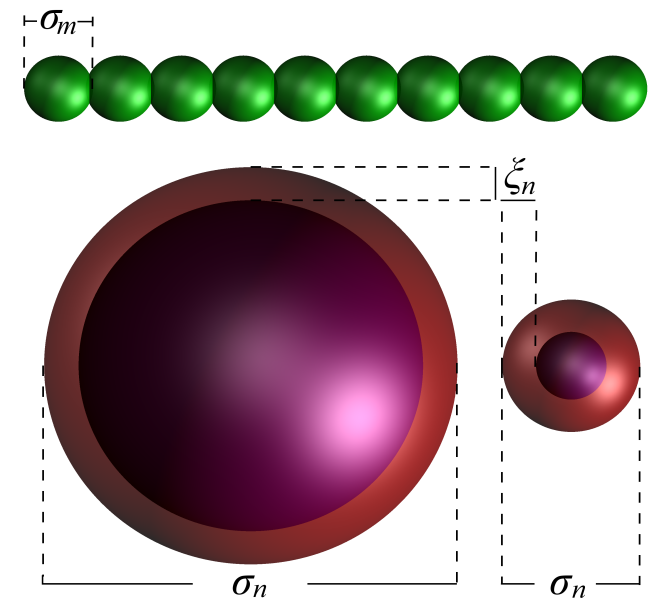

FIG. 1. Schematic representation of the model polymer chains and NPs employed in this work. The hard core and soft shell of the NPs are shown in purple and semi transparent red, respectively, whereas the polymer beads are shown in green. $\sigma_{m}$ and $\sigma_{n}$ are the diameters of the chain bead and NP, respectively. $\xi_{n}$ is the NP's shell thickness. This figure has been generated with the Visual Molecular Dynamics (VMD) software [56].

$$
V_{F E N E}(r)=-\frac{1}{2} K R_{0}^{2} \ln \left(1-\left(r / R_{0}\right)^{2}\right)
$$

where $K /\left(\epsilon / \sigma_{m}^{2}\right)=30$ and $R_{0} / \sigma_{m}=1.5$ are the intensity and the range of the interaction, respectively [54]. Therefore, the whole interaction between adjacent beads results from the application of two contributions:

$$
U_{\text {bonding }}(r)=V_{F E N E}(r)+U^{m m, a d j}(r)
$$

where the superscript $a d j$ indicates that only adjacent beads in a chain are affected. The minimum value of $U_{\text {bonding }}$ is located at $R_{e q}^{m m, a d j} / \sigma_{m} \simeq 0.960897$, and the bonding length assumes values in the range $0<r / \sigma_{m}<1.5$. The value for $R_{e q}^{m m, a d j}$ was obtained by performing numerical computation on Mathematica [57].

\section{METHODOLOGY}

All the MD simulations have been performed in the large scale atomic/molecular massively parallel simulator (LAMMPS) molecular dynamics package [58], with a timestep length $\delta t / \tau=10^{-3}$, where $\tau=M_{m}^{1 / 2} \sigma_{m} \epsilon^{-1 / 2}$ is the system's time unit. Structural, dynamic, rheological, and thermal properties of PNCs have been investigated in the the canonical (NVT) ensemble, where $T$ is the absolute temperature and $V$ the volume of a cubic simulation box with periodic boundaries. We applied a Nosé-Hoover thermostat to set the velocity of the beads and hence the kinetic energy of the system in order to keep the temperature at the constant value of $T=\epsilon / k_{B}$, with $k_{B}=1.38 \times 10^{-23} \mathrm{~J} \mathrm{~K}^{-1}$ the Boltzmann constant. The details of the set up and equilibration processes can be found in the Supporting Information.

After equilibration, the structural properties were assessed by determining the radial distribution functions (RDFs), the polymer radius of gyration $\left(R_{g}\right)$, the end-to-end distance $\left(R_{e e}\right)$, and the interparticle distance $(I D)$. Due to the size polydispersity of NPs, a more appropriate RDF has been calculated by considering a surface-to-surface distance, rather than the usual distance between centers of mass. To this end, the NP/NP RDF must take into account the NP hard cores' radii as follows:

$$
g_{n n}^{\prime}(r)=\frac{1}{\sum_{j=1}^{N_{n_{j}}} \rho_{j}}\left\langle\frac{N_{n_{j}}\left(r-R^{n_{i} n_{j}}\right)}{v(r)}\right\rangle_{\forall n_{i}},
$$

where $N_{n_{j}}$ is the number of particles $j$ with diameter $\sigma_{n_{j}}$ at a surface-to-surface distance $r-R^{n_{i} n_{j}} \pm \Delta r / 2$ from particles $i$ of diameter $\sigma_{n_{i}}, \rho_{j}$ their number density, $v(r)$ the volume of the spherical shell of radius $r \pm \Delta r / 2$, which is concentric to the particles $i$ and whose thickness is $\Delta r / \sigma_{m}=0.1$.

The structure of the polymer chains was investigated by computing the ensemble averaged radius of gyration, $R_{g} \equiv$ $\left\langle R_{g}^{2}\right\rangle^{1 / 2}$, and the end-to-end distance, $R_{e e} \equiv\left\langle R_{e e}^{2}\right\rangle^{1 / 2}$. The former reads [59]

$$
\left\langle R_{g}^{2}\right\rangle=\frac{1}{N_{c}^{2}} \sum_{i=1}^{L_{c}} \sum_{j=i}^{L_{c}}\left\langle\left(\mathbf{R}_{i}-\mathbf{R}_{j}\right)^{2}\right\rangle
$$

where $\mathbf{R}_{i}$ and $\mathbf{R}_{j}$ are the position vectors of beads $i$ and $j$, respectively. The end-to-end distance is the sum of all the bonds making up a polymer chain. A random distribution of chains, like in an isotropic melt, would provide a zero average. Therefore, a mean-squared end-to-end distance is calculated:

$$
\left\langle R_{e e}^{2}\right\rangle=\sum_{i=1}^{L_{c}-1} \sum_{j=1}^{L_{c}-1}\left\langle\mathbf{l}_{i} \cdot \mathbf{l}_{j}\right\rangle
$$

where $\mathbf{l}_{i}=\mathbf{R}_{i+1}-\mathbf{R}_{i}$ is the bond vector connecting two consecutive chain beads.

Additionally, the interparticle distance, $I D$, is a function of the NP's geometry, polydispersity and concentration, and provides a theoretical prediction of the average distance between particles that are randomly distributed in a volume. It reads [49]

$$
I D\left(\left\langle\sigma_{n}\right\rangle, \Pi_{Ð}, \phi_{n}\right)=\left\langle\sigma_{n}\right\rangle\left[\left(\frac{2}{\pi \phi_{n}}\right)^{1 / 3}\left(1+\Pi_{Ð}\right)-1\right],
$$

where the factor $2 / \pi \simeq 0.64$ is the random close packing of spheres. 
To assess the mobility of both polymer chains and NPs, we determined the mean square displacement, $M S D(t) \equiv$ $\left\langle\left|\mathbf{R}(t)-\mathbf{R}\left(t_{0}\right)\right|^{2}\right\rangle$-with $\mathbf{R}(t)$ the position at time $t>t_{0}, \mathbf{R}\left(t_{0}\right)$ the position at time $t_{0}$, and $t_{0}$ the initial time- , and the diffusion coefficients at long time scales. The diffusion coefficient of large spherical particles in a liquid is generally well predicted by the Stokes-Einstein relation, which reads [60]

$$
D_{n}^{S E}=\frac{k_{B} T}{6 \pi \eta_{0} R_{H}},
$$

where $\eta_{0}$ is the solvent viscosity and $R_{H} \simeq \sigma_{n} / 2$ the hydrodynamic radius of the particle. In unentangled polymer melts, the validity of Eq. (15) has been corroborated for particles with diameter $\sigma_{n} \gg 2 R_{g}$. However, the particles in our model polymer melt are comparable in size with the polymer's radius of gyration and, therefore, Eq. (15) is not expected to accurately reproduce their diffusivity [17]. Therefore, to compare our simulation results with more precise theoretical predictions, we referred to three models that explicitly incorporate the effect of a characteristic length of the medium, namely the polymer radius of gyration $[18,20]$ or the monomer size $[15,17]$. In particular, the model proposed by Ganesan et al. modifies Eq. (15) by introducing an additional term reflecting the non-continuous nature of the solvent [18]:

$$
D_{n}^{G}=\frac{k_{B} T}{6 \pi \eta_{0}}\left[\frac{1+3 \lambda}{1+2 \lambda} \frac{1}{\sigma_{n} / 2}+\frac{6 \lambda}{1+2 \lambda} \frac{\left(2 R_{g}\right)^{2}}{\sigma_{n}^{3} / 2}\right],
$$

where $\lambda$ is an adjustable parameter that ranges from 0 , for perfectly stick boundary conditions (bc), to $\infty$, for perfectly slip bc. Similar dependences on the NP and polymer geometry are also found in the model proposed by Yamamoto and Schweizer [20]:

$$
D_{n}^{Y S}=\frac{k_{B} T}{6 \pi \eta_{0}}\left[\frac{1}{\sigma_{n} / 2}+\frac{9}{4} \frac{\left(2 R_{g}\right)^{2}}{\sigma_{n}^{3} / 2}\right] .
$$

By contrast, Liu et al., inspired by the model proposed by Ould-Kaddour and Levesque for diffusion of NPs in simple liquids [15], proposed a slight modification of Eq. (15), taking into account the size of the polymer bead rather than the radius of gyration [17]:

$$
D_{n}^{O L}=\frac{k_{B} T}{6 \pi \eta_{0} \bar{\sigma}_{n_{i} m}} .
$$

In the above equation, $R_{H}$ is substituted with an effective radius $\bar{\sigma}_{n_{i} m}=\left(\sigma_{n}+\sigma_{m}\right) / 2$ to take into account the minimum distance between particles and solvent molecules, being nonzero in a non-continuous medium and increasing the effective size of the particle.

It should be noticed that these theories describe the diffusivity of isolated particles or, equivalently, particles at very dilute concentrations in a medium. In order to consistently compare our simulation results with the predictions of these theoretical models, the polymer melts investigated here embody NPs at the volume fraction $\phi_{n}=0.05$, which is expected to be low enough to consider their mutual interactions of secondary relevance with respect to the $\mathrm{NP} /$ polymer interactions. Additionally, the simulation of a polymer melt containing polydisperse NPs provides average diffusion coefficients that should be consistently compared with the above mentioned theories and, more generally, with any theory describing the diffusivity of particles of a given size in a medium. These diffusion coefficients read

$$
\left\langle D_{n}\right\rangle=\frac{1}{\zeta \sqrt{2 \pi}} \int_{0}^{\infty} \frac{\mathrm{d} \sigma_{n}}{\sigma_{n}} D_{n} e^{-\frac{\left(\ln \sigma_{n}-\mu\right)^{2}}{2 \zeta^{2}}}
$$

and

$$
\left\langle D_{n}\right\rangle_{\sigma_{c}}=\frac{1}{\Omega} \frac{1}{\zeta \sqrt{2 \pi}} \int_{\sigma_{c}}^{\infty} \frac{\mathrm{d} \sigma_{n}}{\sigma_{n}} D_{n} e^{-\frac{\left(\ln \sigma_{n}-\mu\right)^{2}}{2 \zeta^{2}}},
$$

where $\left\langle D_{n}\right\rangle_{\sigma_{c}}$ and $\left\langle D_{n}\right\rangle$ are the diffusivities of the NPs as averaged with the pdfs given in Eqs. (1) and (2), respectively. Therefore, to evaluate the effect of the size dispersity on the NP diffusivity, we plugged Eqs. (15) to (18) into Eqs. (19) and (20).

Finally, the shear viscosity, $\eta$, and thermal conductivity, $\kappa$, of our model PNCs were calculated by using Reverse NonEquilibrium Molecular Dynamics (RNEMD), originally proposed by Müller-Plathe for LJ fluids [61-63] and later extended to polymers [64]. This method allows to compute the above mentioned transport properties by swapping velocities (velocity components or absolute speeds to determine viscosity or thermal conductivity, respectively) between beads at one side of the simulation box and beads located at its center, thus inducing a shear or temperature gradient caused by a known and controlled velocity flux. This flux, in the low perturbation regime, is proportional to the gradient previously induced with proportionality constants $\eta$, for shear gradients, or $\kappa$, for temperature gradients. Additional details are provided in the Supplemental Information.

The finite size effects should not affect too significantly the outcome of our simulations. With regard to the NP diffusivities, the hydrodynamic drag produced by a particle's images in very dilute solutions may be large $[65,66]$. However, if the systems are not infinitely diluted, the effects of hydrodynamic drag due to surrounding particles are counterbalanced by two additional effects. Firstly, an enhancement in mobility is caused by fluctuations of the particle number density, which overcomes the hydrodynamic drag produced by neighboring particles [67]. Secondly, the existence of a partial hydrodynamic screening caused by the neighboring particles can weaken the effects of self-images on the diffusivities [68, 69]. Concerning the finite size effects on the transport properties, they are expected to be negligible on both viscosity [66] and thermal conductivity [70], for the simulation box sizes used in this study [70]. 


\section{RESULTS AND DISCUSSION}

The aim of the present study is to assess the impact of NP size dispersity on the microscopic and macroscopic properties of model polymer nanocomposites. To this end, in this section, we first analyze the dispersion of small and large polydisperse NPs in a dense polymer melt; then present our theoretical framework providing the diffusivity of NPs as a function of their size dispersity in the melt and validate it with simulations; and finally evaluate the shear viscosity and thermal conductivity of a polymer incorporating polydisperse NPs.

\section{Dispersion of NPs in the polymer melt}

Two typical configurations of the model PNCs studied in this work are shown in Fig. 2 for systems containing 30 monodisperse (left frame) and 30 polydisperse (right frame) NPs. As observed in previous works [30, 71], the conformation of the polymer chains is not significantly affected by the presence of NPs and both the radius of gyration and endto-end distance keep a roughly constant value. In particular, $R_{g} / \sigma_{m}=1.49 \pm 0.24$ and $R_{e e} / \sigma_{m}=3.52 \pm 1.17$, with $R_{e e}^{2} / R_{g}^{2} \simeq 5.6$, which indicates, to good approximation, a random coil behavior of the polymer chains.
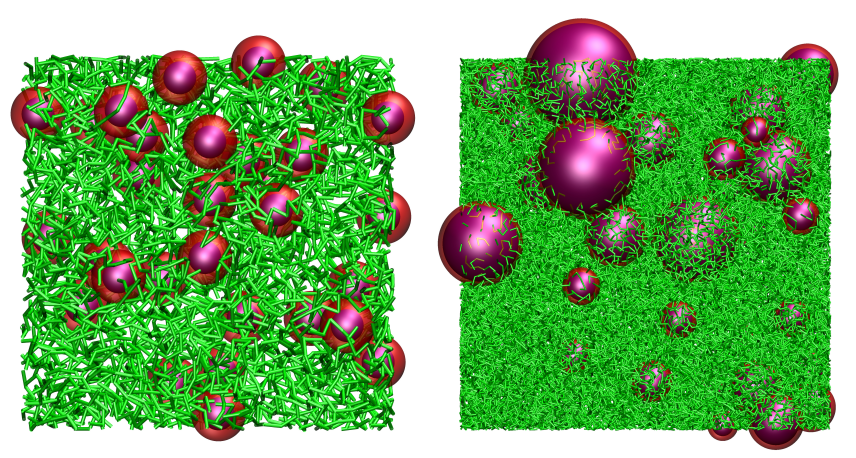

FIG. 2. Representative snapshots of a polymer melt incorporating 30 monodisperse (left frame, $\sigma_{n} / \sigma_{m}=3$ ) and 30 polydisperse (right frame, $\left.\left\langle\sigma_{n}\right\rangle / \sigma_{m}=6, \Pi_{D}=0.20\right) \mathrm{NPs}$ in simulation boxes of size $L / \sigma_{m}=20.4$ and 40.9 , respectively. The number of polymer chains, which are shown in green and reduced in size for clarity, is 564 (left) and 6863 (right). The hard core and soft shell of the NPs are shown in purple and semi transparent red, respectively. Both snapshots have been generated with the Visual Molecular Dynamics (VMD) software [56].

The distribution of particles in the simulation box appears to be rather homogeneous in both cases, with the polymer chains organized evenly. As a matter of fact, the NP surfaceto-surface radial distribution functions shown in Fig. 3 for different size dispersities and average diameters, locate the NPs at the mutual distance $d=\left(r-R^{n_{i} n_{j}}\right) / \sigma_{m} \geqslant 2$, which prevents their clustering and promotes the intercalation of a monolayer of polymer chains in between them. In general, polydispersity and average size do not influence significantly the distri- bution of the NPs in the melt. However, a secondary peak at distances $\left(r-R^{n_{i} n_{j}}\right) / \sigma_{m} \simeq 1.1$ emerges when the average NP diameter decreases to $\left\langle\sigma_{n}\right\rangle / 2 R_{g}=2 / 3$ (Fig. 3d). In this case, a slight tendency of NPs to approach each other and eventually form aggregates can be appreciated. To determine which set of NPs with diameter $\left\langle\sigma_{n}\right\rangle / 2 R_{g}=2 / 3$ might predominantly contribute to the formation of this secondary peak, we calculated the $g_{n_{i} n_{j}}^{\prime}(r)$ of monodisperse NPs with diameter $\sigma_{n} / 2 R_{g}=1 / 3$ and detected a more substantial, but not dominant, tendency towards clustering with a peak also located at $\left(r-R^{n_{i} n_{j}}\right) / \sigma_{m} \simeq 1.1$. We therefore conclude that very small NPs, namely NPs with diameter $\sigma_{n} / 2 R_{g} \leq 2 / 3$, have a larger probability to form aggregates as compared to bigger NPs.

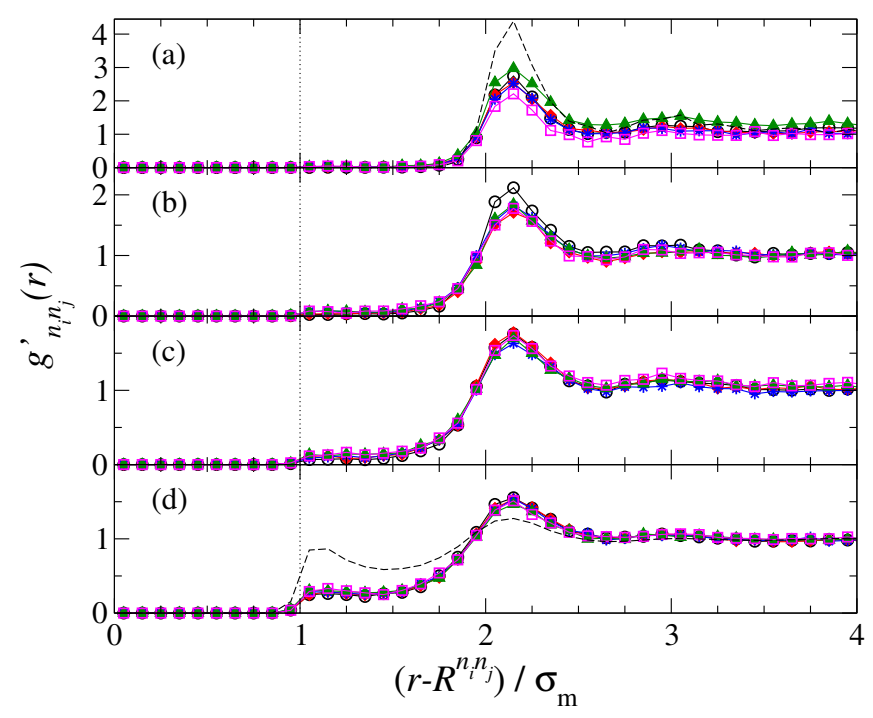

FIG. 3. Surface-to-surface radial distribution functions, $g_{n_{i} n_{j}}^{\prime}(r)$, of NPs with average diameter $\left\langle\sigma_{n}\right\rangle / 2 R_{g}=2$ (a), 4/3 (b), 1 (c), and $2 / 3(\mathrm{~d})$, and size polydispersity $\Pi_{D}=0.00(\bigcirc), \Pi_{D}=0.05(\diamond)$, $\Pi_{D}=0.10(*), \Pi_{D}=0.15(\Delta)$, and $\Pi_{D}=0.20(\square)$. For comparison, the $g_{n_{i} n_{j}}^{\prime}(r)$ of monodisperse NPs with $\sigma_{n} / \sigma_{m}=8 / 3$ and $1 / 3$ are shown as dashed lines in frames (a) and (d), respectively. The vertical dotted line indicates the value of the contact distance, $R^{n_{i} n_{j}}+\sigma_{m}$, between two NPs.

To interpret this result, the interparticle distance should be compared to the characteristic length governing the relaxation dynamics of unentangled polymer chains, for instance the polymer radius of gyration, see Fig. 4. In particular, $I D / 2 R_{g} \leqslant 1$ in those systems containing NPs with diameter $\left\langle\sigma_{n}\right\rangle / 2 R_{g} \leqslant 2 / 3$, but larger in the remaining systems. Given that the NP/polymer interactions are kept constant across the systems studied, we conclude that the tendency to aggregate of the smallest NPs must be promoted by entropic contributions that maximize the available volume for the polymer chains and overcome the NP-NP repulsion, which has an enthalpic origin. This scenario is not observed in systems where $I D / 2 R_{g}>1$, at which the mobility of chains between NPs is not particularly hampered. 


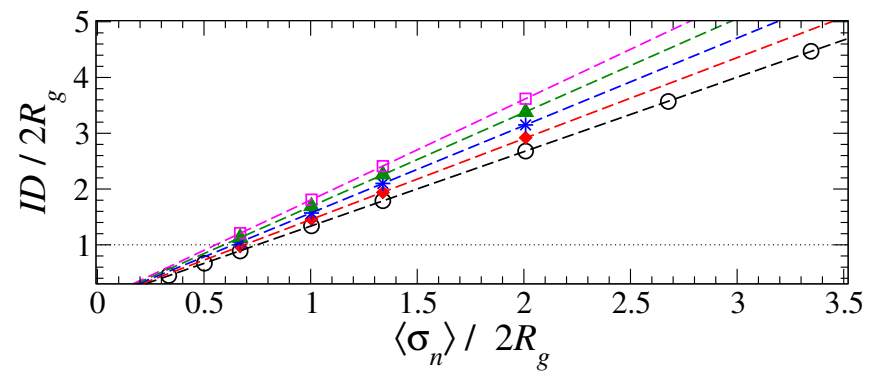

FIG. 4. Interparticle distance as a function of the average NP diameter, as obtained from Eq. (14) for different values of polydispersity and constant $\phi_{n}=0.05$. The symbols indicate the systems studied in this work, with $\Pi_{\ni}=0.00(\bigcirc), 0.05(\diamond), 0.10(*), 0.15(\Delta)$, and $0.20(\square)$. The dotted line represents the value $I D=2 R_{g}$.

\section{Diffusion of NPs in the polymer melt}

\section{Theoretical predictions}

Our model polymer melts incorporate polydisperse NPs with average diameter $\left\langle\sigma_{n}\right\rangle$. In order to obtain a functional dependence of their average diffusivity on the polydispersity index, we assume that the NPs are entirely surrounded by polymer chains and do not feel the presence of other NPs in the melt. This assumption, which implies a dilute concentration of well-dispersed and weakly interacting NPs in the polymer, is supported by the results shown in Fig. 3 and in agreement with the typical formulations of most PNCs. Therefore, the average value of the NP diffusion coefficient, $\left\langle D_{n}\right\rangle$, as obtained by Eq. (19), was used to include the effect of size dispersity in Eqs. (15) to (18). In particular, the SE relation and the models proposed by Ganesan et al. and Yamamoto and Schweizer were straightaway adapted by substituting $\sigma_{n}^{-1}$ and $\sigma_{n}^{-3}$ in Eqs. (15) to (17) with the moments calculated from Eq. (6) with $N=-1$ and $N=-3$, respectively. This modification provides the following three average diffusion coefficients:

$$
\left\langle D_{n}^{S E}\right\rangle=\frac{k_{B} T\left(1+\Pi_{Ð}\right)}{6 \pi \eta_{0}\left\langle\sigma_{n}\right\rangle / 2}
$$

$$
\left\langle D_{n}^{G}\right\rangle=\frac{k_{B} T}{6 \pi \eta_{0}}\left[\frac{1+3 \lambda}{1+2 \lambda} \frac{1+\Pi_{Ð}}{\left\langle\sigma_{n}\right\rangle / 2}+\frac{6 \lambda}{1+2 \lambda} \frac{\left(2 R_{g}\right)^{2}\left(1+\Pi_{\oplus}\right)^{6}}{\left\langle\sigma_{n}\right\rangle^{3} / 2}\right],
$$

$$
\left\langle D_{n}^{Y S}\right\rangle=\frac{k_{B} T}{6 \pi \eta_{0}}\left[\frac{1+\Pi_{D}}{\left\langle\sigma_{n}\right\rangle / 2}+\frac{9}{4} \frac{\left(2 R_{g}\right)^{2}\left(1+\Pi_{\oplus}\right)^{6}}{\left\langle\sigma_{n}\right\rangle^{3} / 2}\right] .
$$

By contrast, to estimate the average diffusion coefficient within the model by Ould-Kaddour and Levesque, Eq. (18), we expanded the effective radius, $\bar{\sigma}_{n_{i} m}=\left(\sigma_{n}+\sigma_{m}\right) / 2$, in Taylor series and obtained the following convergent infinite summation:

$$
\begin{array}{r}
\left\langle D_{n}^{O L}\right\rangle=\frac{k_{B} T\left(1+\Pi_{D}\right)}{6 \pi \eta_{0}\left\langle\sigma_{n}\right\rangle}\left\{\operatorname{erfc}\left(\frac{\ln \left(\frac{\sigma_{m}}{\left\langle\sigma_{n}\right\rangle}\right.}{\sqrt{2 \ln \left(1+\Pi_{D}\right)}}\right)+\right. \\
+\sum_{k=1}^{\infty}(-1)^{k}\left[-\left(\frac{\left\langle\sigma_{n}\right\rangle}{\sigma_{m}}\right)^{k / 2}\left(1+\Pi_{D}\right)^{k(k-3) / 2} \operatorname{erfc}\left(\frac{\ln \left(\frac{\left\langle\sigma_{n}\right\rangle}{\sigma_{m}}\left(1+\Pi_{D}\right)^{k-3 / 2}\right)}{\sqrt{2 \ln \left(1+\Pi_{D}\right)}}\right)+\right. \\
\left.\left.+\left(\frac{\sigma_{m}}{\left\langle\sigma_{n}\right\rangle}\right)^{k}\left(1+\Pi_{D}\right)^{k(k+3) / 2} \operatorname{erfc}\left(\frac{\ln \left(\frac{\sigma_{m}}{\left\langle\sigma_{n}\right\rangle}\left(1+\Pi_{D}\right)^{k+3 / 2}\right)}{\sqrt{2 \ln \left(1+\Pi_{D}\right)}}\right)\right]\right\},
\end{array}
$$

where $\operatorname{erfc}(x)$ is the complementary error function and $k$ the summation index. The value of $\left\langle D_{n}^{S E}\right\rangle$ is recovered in the limit $\sigma_{m} /\left\langle\sigma_{n}\right\rangle \rightarrow 0$ for finite positive values of $\Pi_{\ni}$. While $\left\langle D_{n}^{S E}\right\rangle$ displays a simple linear dependence on $\Pi_{D}$, the diffusivities $\left\langle D_{n}^{G}\right\rangle$ and $\left\langle D_{n}^{Y S}\right\rangle$ unveil a significantly stronger dependence on $\Pi_{Ð}$, provided by the non-hydrodynamic contribution. Nevertheless, in the so-called colloid regime, where the polymer radius of gyration is much smaller than the particle size $\left(R_{g} \ll \sigma_{n_{i}}\right)$ and the polymer itself can be regarded as a continuous medium, the non-hydrodynamic contributions can be safely neglected, and the diffusion coefficients $\left\langle D_{n}^{G}\right\rangle$ and $\left\langle D_{n}^{Y S}\right\rangle$ change linearly with $\Pi_{Ð}$, as expected. By contrast, in the nanoparticle limit, where the characteristic length scale of the polymer is comparable or larger than the NP size, modeling the polymer in terms of an effective mean-field hydrodynamic interaction would significantly underestimate the NP diffusivity, especially for highly polydisperse NPs. In this case, the non-hydrodynamic contribution that is incorporated in Eqs. (22) and (23) is crucial to assess the mobility of a dilute dispersion of size dispersed NPs. As far as Eq. (24) is concerned, in the range of values of $\left\langle\sigma_{n}\right\rangle$ and $\Pi_{D}$ relevant to our systems, the average diffusivity can be approximated by a power law function of the type $\left\langle D_{n}^{O L}\right\rangle \sim\left(1+\Pi_{D}\right)^{\alpha}$, with fitting exponent $\alpha<1$. Therefore, the model by Ould-Kaddour and Levesque predicts a weaker dependence of the diffusion coefficient on the NP size dispersity as compared to the SE model. These theoretical predictions can be straightaway compared with our simulation results. Nevertheless, to better assess their 
validity in the context of a more realistic scenario, it is convenient to average the diffusion coefficients as obtained by Eqs.
(15) to (18) according to the modified LN distribution that omits NP diameters smaller than the polymer chain bead. In particular, we obtain:

$$
\begin{gathered}
\left\langle D_{n}^{S E}\right\rangle_{\sigma_{c}}=\frac{1}{\Omega} \frac{k_{B} T\left(1+\Pi_{\oplus}\right)}{6 \pi \eta_{0}\left\langle\sigma_{n}\right\rangle} \operatorname{erfc}\left(\frac{\ln \left(\sigma_{c}\left(1+\Pi_{D}\right)^{3 / 2} /\left\langle\sigma_{n}\right\rangle\right)}{\sqrt{2 \ln \left(1+\Pi_{D}\right)}}\right), \\
\left\langle D_{n}^{G}\right\rangle_{\sigma_{c}}=\frac{1}{\Omega} \frac{k_{B} T}{6 \pi \eta_{0}}\left[\frac{1+3 \lambda}{1+2 \lambda} \frac{1+\Pi_{D}}{\left\langle\sigma_{n}\right\rangle} \operatorname{erfc}\left(\frac{\ln \left(\sigma_{c}\left(1+\Pi_{D}\right)^{3 / 2} /\left\langle\sigma_{n}\right\rangle\right)}{\sqrt{2 \ln \left(1+\Pi_{D}\right)}}\right)+\frac{6 \lambda}{1+2 \lambda} \frac{\left(2 R_{g}\right)^{2}\left(1+\Pi_{D}\right)^{6}}{\left\langle\sigma_{n}\right\rangle^{3}} \operatorname{erfc}\left(\frac{\ln \left(\sigma_{c}\left(1+\Pi_{D}\right)^{7 / 2} /\left\langle\sigma_{n}\right\rangle\right)}{\sqrt{2 \ln \left(1+\Pi_{D}\right)}}\right)\right], \\
\left\langle D_{n}^{Y S}\right\rangle_{\sigma_{c}}=\frac{1}{\Omega} \frac{k_{B} T}{6 \pi \eta_{0}}\left[\frac{1+\Pi_{D}}{\left\langle\sigma_{n}\right\rangle} \operatorname{erfc}\left(\frac{\ln \left(\sigma_{c}\left(1+\Pi_{D}\right)^{3 / 2} /\left\langle\sigma_{n}\right\rangle\right)}{\sqrt{2 \ln \left(1+\Pi_{D}\right)}}\right)+\frac{9}{4} \frac{\left(2 R_{g}\right)^{2}\left(1+\Pi_{D}\right)^{6}}{\left\langle\sigma_{n}\right\rangle^{3}} \operatorname{erfc}\left(\frac{\ln \left(\sigma_{c}\left(1+\Pi_{D}\right)^{7 / 2} /\left\langle\sigma_{n}\right\rangle\right)}{\sqrt{2 \ln \left(1+\Pi_{D}\right)}}\right)\right]
\end{gathered}
$$

and

$$
\left\langle D_{n}^{O L}\right\rangle_{\sigma_{c} / \sigma_{m} \geqslant 1}=\frac{1}{\Omega} \frac{k_{B} T\left(1+\Pi_{D}\right)}{6 \pi \eta_{0}\left\langle\sigma_{n}\right\rangle} \sum_{k=0}^{\infty}\left(-\frac{\sigma_{m}}{\left\langle\sigma_{n}\right\rangle}\right)^{k}\left(1+\Pi_{D}\right)^{k(k+3) / 2} \operatorname{erfc}\left(\frac{\ln \left(\frac{\sigma_{c}}{\left\langle\sigma_{n}\right\rangle}\left(1+\Pi_{D}\right)^{k+3 / 2}\right)}{\sqrt{2 \ln \left(1+\Pi_{D}\right)}}\right) .
$$

It should be noticed that, since the function $\operatorname{erfc}(x) / 2<1$, the diffusion coefficients obtained by introducing the cutoff diameter are such that $\left\langle D_{n}\right\rangle_{\sigma_{c}}\left\langle\left\langle D_{n}\right\rangle\right.$ for the same values of $\left\langle\sigma_{n}\right\rangle$ and $\Pi_{D}$. Additionally, while Eqs. (25) to (27) can be applied to $\sigma_{c}>0$, Eq. (28) is only valid for $\sigma_{c} / \sigma_{m} \geqslant 1$. The derivation of Eq. (28) for the case $\left\langle D_{n}^{O L}\right\rangle_{\sigma_{c} / \sigma_{m}<1}$ is provided in the supplemental information.

\section{Simulation results}

To validate our theoretical predictions, we performed MD simulations of polydisperse NPs embedded in a melt of unentangled polymer chains. In particular, we estimated the diffusion coefficients of a wide range of NPs with different average diameter and size dispersity. To this end, we calculated the slope of the NP mean square displacement, $\Delta \mathbf{r}$, at long times and obtained the isotropic average diffusion coefficients $\left\langle D_{n}\right\rangle=\partial\left\langle\Delta \mathbf{r}^{2}\right\rangle / 6 \partial t$. In the following, we compare the simulation results with the theoretical predictions of the modified Stokes-Einstein and Ganesan models, and refer the reader to the supplemental information for the comparison with the $Y S$ and $O L$ models. The diffusion coefficients as predicted by theory and calculated by simulations are shown in in Figs. 5 and 6.

In both figures, the simulated diffusion coefficients (symbols) are shown as a function of the average diameter (frames $a$ and $c$ ) and polydispersity index ( $b$ and $d$ ). More specifically, in Fig. 5, they are compared with the predictions of the modified Stokes-Einstein relations of Eqs. (21) and (25), displayed as dashed lines in the top and bottom frames, respectively.
As expected, $\left\langle D_{n}\right\rangle$ decreases significantly with $\left\langle\sigma_{n}\right\rangle$ as large NPs have a reduced mobility in a dense melt as compared to small NPs. The modified SE theory predicts very well this tendency, regardless of the fact that a cutoff in the size distribution is applied (frame $c$ ) or not (frame $a$ ). The agreement between theory and simulations, which is especially good for monodisperse NPs, it is generally very satisfactory for polydisperse NPs, with deviations no larger than $15 \%$. The original SE theory is able to describe the diffusion of micrometric sized particles in a solvent, but underestimates the diffusion of particles with size in the nanoscale. A similar tendency is also expected when a degree of polydispersity is added. As a matter of fact, the modified SE equation, which predicts a linear growth of the diffusion coefficient with $\Pi_{D}$, displays a gradually decreasing agreement with the simulations at increasing polydispersity (frames $b$ and $d$ ). The theoretical predictions generated by the modified LN distribution are consistent with those obtained with a conventional (without cutoff diameter) LN distribution for all the systems studied here. On the other hand, the theoretical predictions based on Ganesan model and reported in Fig. 6, provide a significantly better agreement with our simulation results, even at large NP size dispersities. The parameter $\lambda=0.011$ in Ganesan model was calculated by fitting the results obtained for monodisperse systems to Eq. (16). This value was then used in Eqs. (22) and (26) to obtain the theoretical predictions for $\Pi_{D}>0$. Since $\lambda \simeq 0$, Ganesan model converges to the Stokes-Einstein relation with stick boundary conditions at large particle diameters.

To understand the effect of NP size dispersity on the mobility of the polymer chains, we investigated the diffusion of the chain beads as a function of the interparticle distance, 


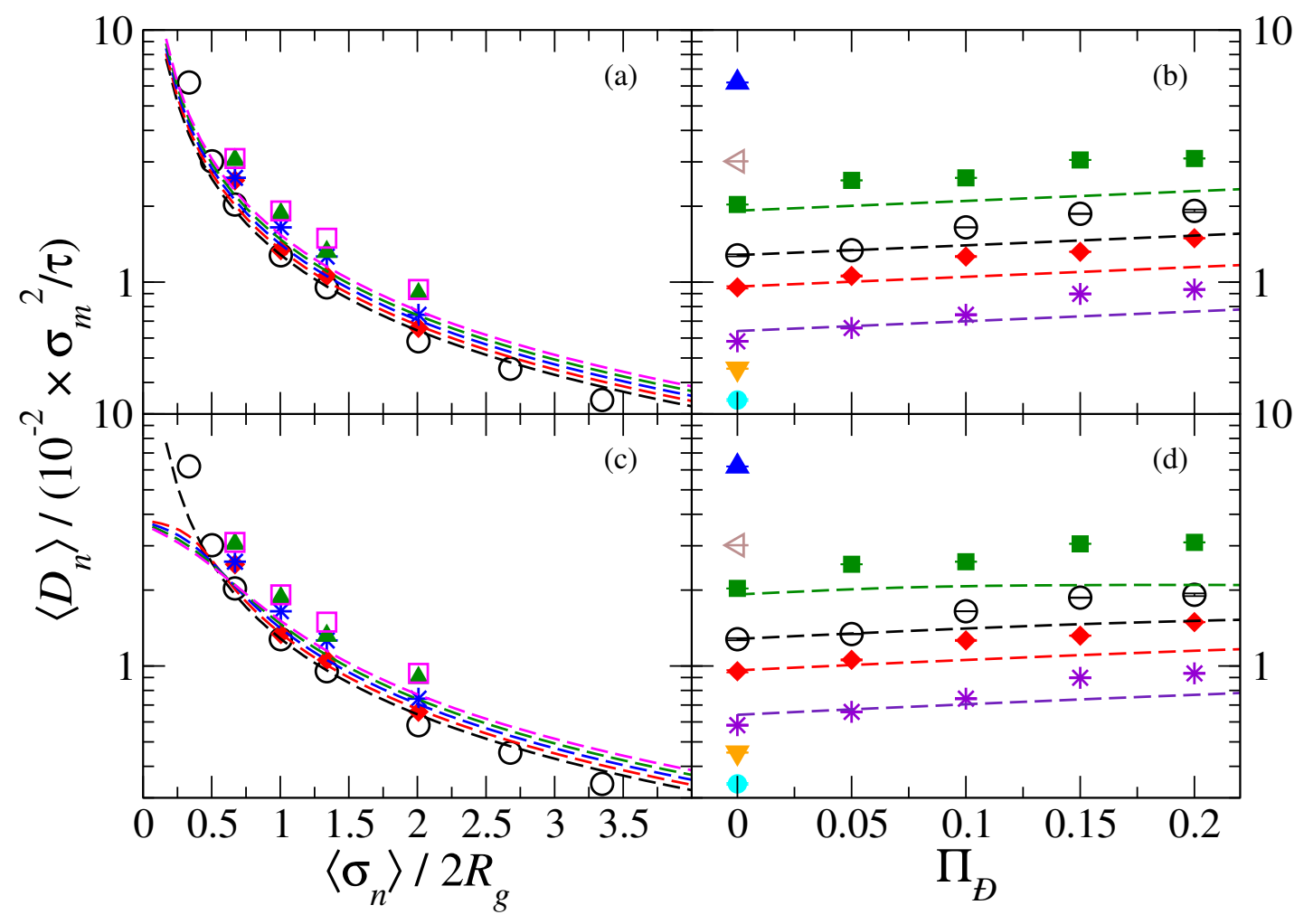

FIG. 5. Average diffusion coefficients of NPs in melts of unentangled polymer chains as a function of the average NP diameter (a, c) and polydispersity index $(\mathrm{b}, \mathrm{d})$. Symbols refer to simulation results, whereas dashed lines to the theoretical predictions of the Stokes-Einstein relation within a Log-normal $(\mathrm{a}, \mathrm{b})$ and modified Log-normal (c, d) size distribution. Left frames: $\Pi_{D}=0.00(\bigcirc), 0.05(\diamond), 0.10(*), 0.15(\Delta)$, and $0.20(\square)$. Right frames: $\left\langle\sigma_{n}\right\rangle / 2 R_{g}=1 / 3(\Delta), 1 / 2(\triangleleft), 2 / 3(\square), 1(\bigcirc), 4 / 3(\diamond), 2(*), 8 / 3(\nabla)$, and 10/3 $(\bigcirc)$. The range of colors used for the dashed lines follows the same description as the symbols. Error bars are smaller than the symbol size and might not be visible.

$I D=I D\left(\left\langle\sigma_{n}\right\rangle, \Pi_{D}, \phi_{n}\right)$. The reduced diffusion coefficient of the chain beads, $D_{m} / D_{m 0}$, where $D_{m 0}$ is calculated in the pure polymer melt, is shown in Fig. 7 for a range of interparticle distances between $R_{g}$ and $9 R_{g}$. We note that increasing the interparticle distance promotes the mobility of the polymer chains. A similar effect is also observed if, for a given NP average diameter, the NP size dispersity increases, as shown by each set of symbols in Fig. 7. Therefore, we can conclude that, by promoting particle separation, polydispersity favors the mobility of the polymer chains and enhances their diffusion coefficients. Recently, Composto et al. studied the tracer diffusion of deuterated polystyrene in a polystyrene nanocomposite incorporating monodisperse or polydisperse NPs [49]. These authors highlighted the existence of an empirical master curve of the type $D_{m} / D_{m 0} \sim \ln \left(I D / 2 R_{g}\right)$, which describes the dependence of the reduced diffusion coefficient on the interparticle distance for a spectrum of PNCs. Our simulation results in Fig. 7 confirm these experimental observations and indicate the existence of a unique master curve predicting the reduced diffusivity of chain beads as a function of $I D$ for all the model PNCs studied here. Our fit to these data is given by $D_{m} / D_{m 0}=2 / \pi \arctan \left(2.845 I D / 2 R_{g}\right)$, with $D_{m} \rightarrow D_{m 0}$ if the interparticle distance is large enough to disregard the effect of the NPs on the diffusivity of the polymer chains, and $D_{m} \rightarrow 0$ if the interparticle distance is so small that the mobility of the polymer chains is completely inhibited.

\section{Viscosity}

The effect of the NP size dispersity on the zero-shear viscosity is shown in the top frame of Fig. 8. In particular, we display the zero-shear viscosity, $\eta$, normalized by the viscosity value obtained for the pure polymer melt, $\eta_{0}$, and compare it with that of a polymer composite incorporating micron or larger particles, that is $\eta_{\mu} \equiv \eta_{0} F\left(\phi_{n}\right)=\eta_{0}\left[1+2.5 \phi_{n}+4.94 \phi_{n}^{2}\right]$ $[23,31]$. More specifically, $F\left(\phi_{n}\right)$ incorporates the effect of large particles via their volume fraction $\phi_{n}$. For clarity, both $\eta_{0}$ and $\eta_{\mu}$ are shown in Fig. 8 with a solid and dotted line, respectively. As a general tendency, polydispersity does not seem to affect the results very significantly. We note a slightly decrease in $\eta$ at increasing polydispersity index, but this is not a remarkable effect. Nevertheless, other interesting observations can be made. At NP diameters significantly larger than the polymer radius of gyration, that is $\sigma_{n} / 2 R_{g} \geq 2$, the viscosity of our model PNCs tends to that of a conventional composite described by the above virial expansion, $\eta_{\mu}$. In other words, the particles are gradually losing their nanoscopic signature in such a way that their effect on the rheology of the polymer can 


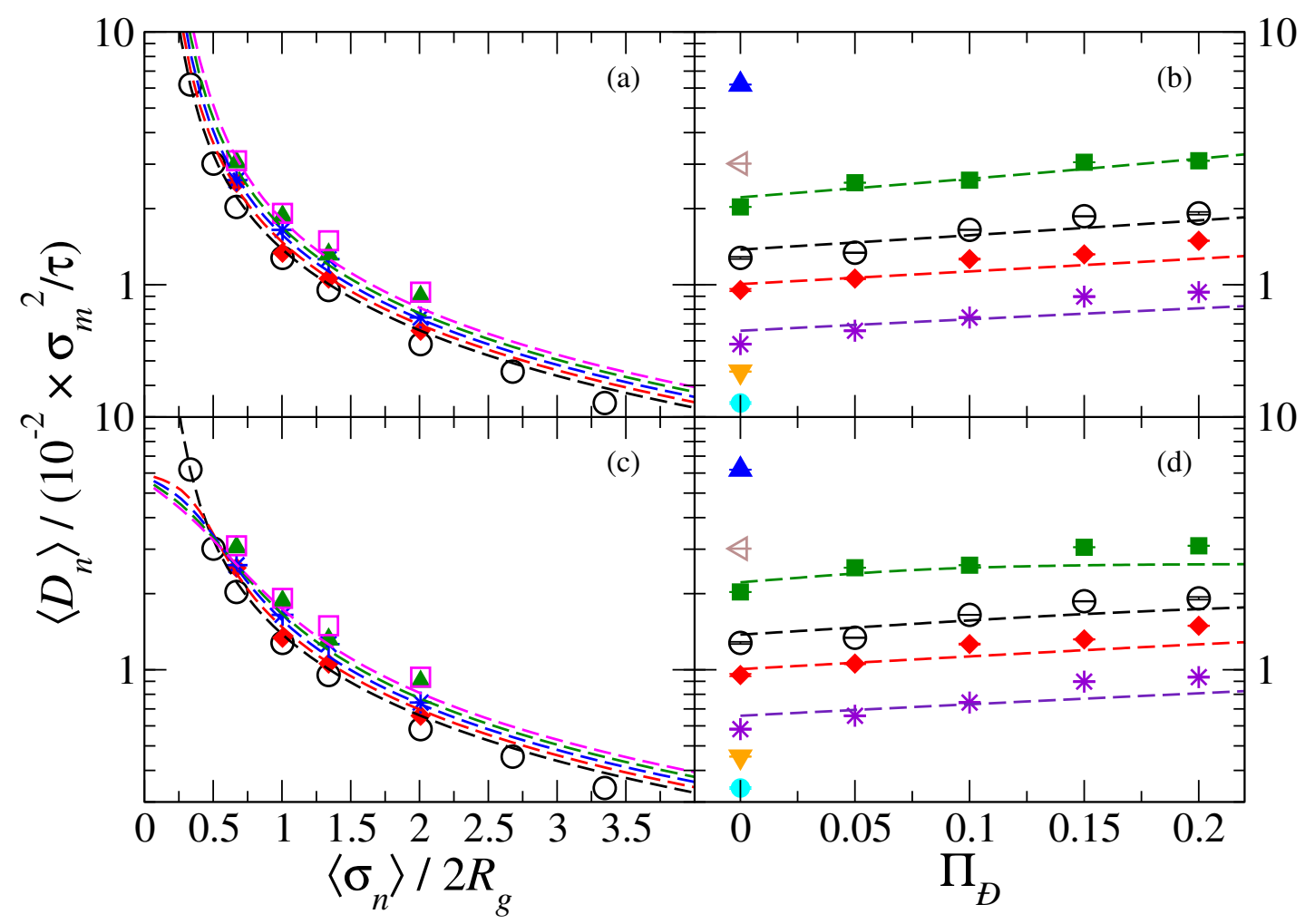

FIG. 6. Average diffusion coefficients of NPs in melts of unentangled polymer chains as a function of the average NP diameter (a, c) and polydispersity index (b, d). Symbols refer to simulation results, whereas dashed lines to the theoretical predictions of the Ganesan's model within a Log-normal (a, b) and modified Log-normal (c, d) size distribution. Left frames: $\Pi_{D}=0.00(\bigcirc), 0.05(\diamond), 0.10(*), 0.15(\Delta)$, and $0.20(\square)$. Right frames: $\left\langle\sigma_{n}\right\rangle / 2 R_{g}=1 / 3(\Delta), 1 / 2(\triangleleft), 2 / 3(\square), 1(\bigcirc), 4 / 3(\diamond), 2(*), 8 / 3(\nabla)$, and 10/3 $(\bigcirc)$. The range of colors used for the dashed lines follows the same description as the symbols. Error bars are smaller than the symbol size and might not be visible.

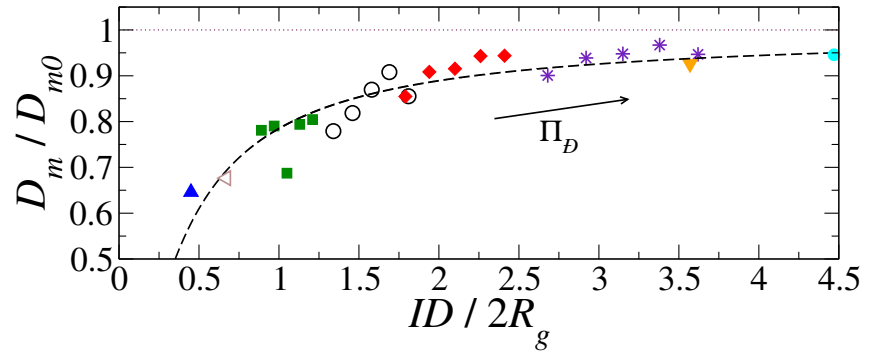

FIG. 7. Reduced diffusion coefficients of the polymer chain beads as a function of the interparticle distance at different NP diameters and size dispersities. Each set of symbols indicates a given NP size and an increasing NP size dispersity from left $\left(\Pi_{D}=0\right)$ to right $\left(\Pi_{D}=0.20\right)$. In particular, $\left\langle\sigma_{n}\right\rangle / 2 R_{g}=1 / 3(\Delta), 1 / 2(\triangleleft), 2 / 3(\square), 1$ $(\bigcirc), 4 / 3(\diamond), 2(*), 8 / 3(\nabla)$, and 10/3 (○). It should be noticed that $\boldsymbol{\Lambda}, \triangleleft, \nabla$, and $\bigcirc$ refer to monodisperse NPs. The dotted and dashed lines are the diffusivity of chain beads in a pure polymer melt and an empirical fit of the type $D_{m} \sim \arctan (I D)$, respectively.

be very well described by their volume fraction only.

By contrast, for particle diameters comparable to or smaller than $R_{g}$, the zero-shear viscosity gradually increases up to $\eta \approx 1.3 \eta_{\mu}$. To obtain the same viscosity with micron-sized particles, a larger particle's volume fraction would be nec- essary, namely $16 \%$ rather than $5 \%$. This result unveils an interesting thickening effect, with the viscosity of the PNCs being larger than that of the pristine polymer and increasing at decreasing NP diameter. Although other works determined a plasticizing effect upon addition of small NPs to a polymer melt $[29,30]$, we stress here that controlling the viscosity of a polymer melt by addition of NPs is a challenging task that depends on a large number of complex parameters, including, but not limited to, the strength of the NP/polymer interaction. Kumar et al. modeled the behavior of polymer melts incorporating either attractive or athermal NPs and observed a change in their viscosity depending on the polymer chain length, NP size and strength of NP/polymer interaction [29]. For attractive NPs with size $\sigma_{n} \geq \sigma_{m}$ in a melt of unentangled chains, these authors observed an increase in the viscosity with respect to that of the polymer melt, that is a thickening effect, in agreement with our results.

The increase in viscosity when decreasing NP size at constant NP volume fraction, can be explained in terms of specific interface area, $s_{v}$, which determines the fraction of chains at the $\mathrm{NP} /$ polymer interface whose dynamics are significantly affected by the interaction with the NPs [31]. The specific interface area reads 

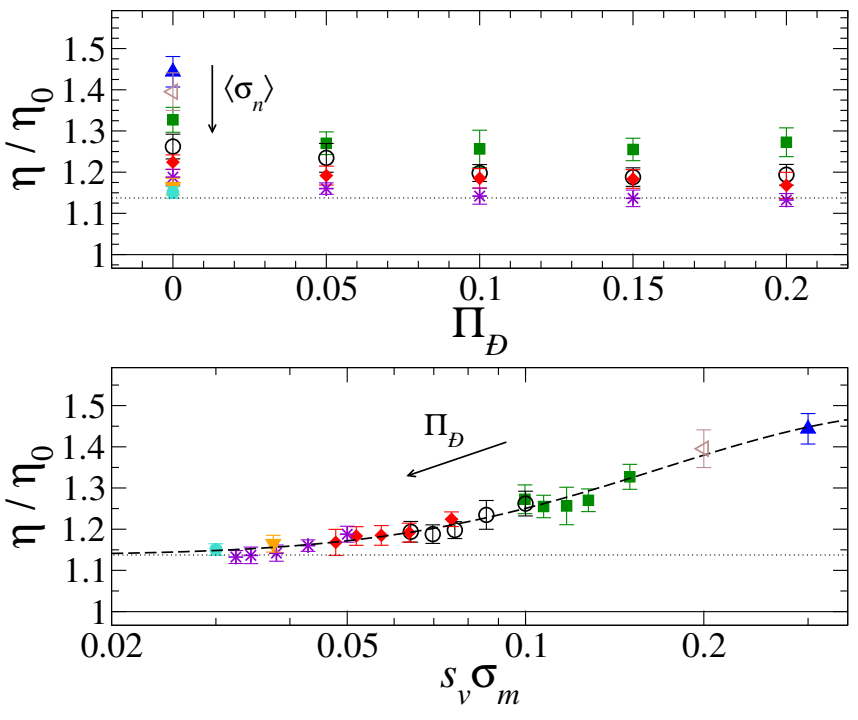

FIG. 8. Zero-shear viscosity, $\eta$, of polymer melts incorporating NPs of different size and size dispersity, normalized with that of the pure polymer melt $\eta_{0}$. Top and bottom frames provide the dependence of $\eta$ on the polydispersity index, $\Pi_{\ni}$, and specific interface area, $s_{v}$, respectively. Symbols refer to simulation results, while the dotted and solid lines indicate the value of $\eta_{\mu}$ and $\eta_{0}$, respectively. The dashed line in the bottom frame is a guide for the eye. NP size: $\left\langle\sigma_{n}\right\rangle / 2 R_{g}=1 / 3(\boldsymbol{\Delta}), 1 / 2(\triangleleft), 2 / 3(\square), 1(\bigcirc), 4 / 3(\diamond), 2(*), 8 / 3(\nabla)$, and $10 / 3(\bigcirc)$.

$$
s_{v}=\frac{6 \phi_{n}}{\left\langle\sigma_{n}\right\rangle\left(1+\Pi_{\oplus}\right)^{2}},
$$

where we observe a dependence on NP size, size dispersity, and volume fraction. All the systems investigated in this work contain a volume fraction of NPs which is kept constant at $\phi_{n}=0.05$. Therefore, the only parameter that can determine any change in $s_{v}$, for the same polydispersity index, is the NP average diameter. In particular, the smaller this diameter, the larger the specific $\mathrm{NP} /$ polymer interface area where the polymer chains, due to the attractive NP/polymer interactions, would preferentially distribute. Keeping this in mind, we now analyze the effect of $s_{v}$ on the viscosity of our model PNCs, which is presented in the bottom frame of Fig. 8. An interesting implication should be highlighted here. Regardless of the NP size and size dispersity, all the results collapse on a single master curve. This very significant result indicates that the viscosity does not depend directly on the NP size or size dispersity, but is very much influenced by the resulting specific area that forms at the NP/polymer interface. The larger this area, the larger the viscosity of a PNC with respect to that of a traditional composite. In particular, the system with NPs of size $\sigma_{n} / 2 R_{g}=1 / 3$ (blue triangle in Fig. 8) shows the largest viscosity, which gradually decreases to that of a polymer melt incorporating large, micron-sized particles. The curve displayed in Fig. 8 (bottom frame) is just a guide for the eye and should not be used to extrapolate the viscosity at large $s_{v}$. Nevertheless, the slight decrease in $\partial \eta / \partial s_{v}$ when $\sigma_{n} / \sigma_{m}=1$ is most probably due to the increase in $g_{n n}^{\prime}$ at contact distance, which is shown in Fig. 3d. The effects of those NP/NP contacts are still not significant in our systems, but must be considered to predict the rheological response of our model PNCs in systems with $\sigma_{n} / \sigma_{n}<1$. However, we want to stress that the incorporation of such small NPs was discarded in order to avoid unrealistic systems, since, in practice, NPs cannot be made as small as the polymer chain monomer.

\section{Thermal Conductivity}

Here we assess the mere effect of NP size and size dispersity on the polymer thermal conductivity, aware of the fact that our CG model, neglecting most of the details determining the heat transfer across the NP/polymer interface, cannot provide a full understanding of the phenomenon, which might definitely be improved by describing the NPs as clusters of elementary beads with specific physical properties, or by more sophisticated models incorporating quantum effects. As a result of our model NPs being described as single interaction sites, we have observed that they behave as perfect insulators with thermal conductivity $\kappa_{n}=0$.
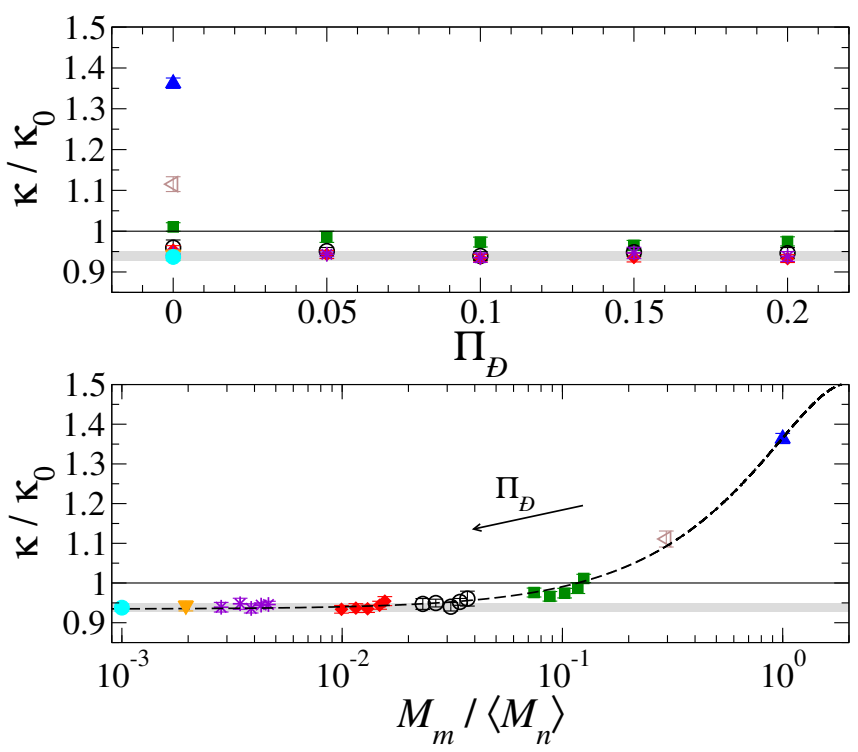

FIG. 9. Thermal conductivity of PNCs incorporating polydisperse NPs as a function of the polydispersity index (top) and inverted average NP mass. NP's diameters: $\left\langle\sigma_{n}\right\rangle / 2 R_{g}=1 / 3(\Delta), 1 / 2(\triangleleft), 2 / 3(\square)$, $1(\bigcirc), 4 / 3(\diamond), 2(*), 8 / 3(\nabla)$, and 10/3 $(\bigcirc)$. The solid lines indicate the thermal conductivity of the pristine polymer melt, $\kappa_{0}$, whereas the shade areas embody the predictions for the large particle limit made by the models mentioned in the text. The dashed line is a guide for the eyes.

In the top frame of Fig. 9, we present the dependence of thermal conductivity on the polydispersity index, which is very weak across the entire range of size dispersities considered in this study. The general tendency observed is that the thermal conductivity measured in our systems decreases as 
compared to that of the pristine polymer, indicated in both frames of Fig. 9 by a solid line, and can be explained as the contribution of the nanofillers' thermal properties to the response of the PNC. Several theories have been proposed to describe the thermal conductivity of composites loaded with micrometric sized particles, all of them describing the heat transfer exclusively in terms of $\phi_{n}, \kappa_{n}$, and the thermal conductivity of the pure polymer melt, $\kappa_{0}$. In particular, the parallel model [37] predicts for our systems a thermal conductivity of $\kappa / \kappa_{0}=0.95$. The Maxwell-Garnett effective medium approximation (MG-EMA) theory [39], Hamilton-Crosser's theory [72], and Nan's model [40], predict for perfect insulators and $\phi_{n}=0.05$ a conductivity value of $\kappa / \kappa_{0}=0.927$, whereas Landauer's relation, obtained from considerations on effective mean percolation theory [73], yields a value of 0.925. Finally, the model proposed by Ordóñez-Miranda and Alvarado-Gil, yields a value of 0.926 , which is NP size independent in the limit $\kappa_{n} \rightarrow 0$ [44]. All these predictions, very close to each other, are represented as a shaded area in each frame of Fig. 9, and are compatible with our results in the large particle limit.

The bottom frame of Fig. 9 displays the values of thermal conductivity as a function of inverted average NP mass. Our results collapse onto a master curve that converges asymptotically to $\kappa / \kappa_{0} \simeq 0.93$ for $M_{m} /\left\langle M_{n}\right\rangle \ll 1$, which confirms the non-conductive nature of our NPs in the large particle limit. By contrast, the reduction in the NP mass causes an abrupt increase in $\kappa$ when particles and polymer chain beads have comparable mass and size. Being our NPs perfect insulators, this intriguing effect appears to be caused by structural differences between the systems containing large and small NPs. A simple theoretical justification to this result can be provided by considering the collisions between pairs of beads, including NPs, in our system. We imagine that the colliding beads are located in contiguous parallel layers, $l_{i}$, with average temperature $T_{i}$. The number of collisions per unit time and layer are proportional to the average number density of beads in each layer, which is temperature dependent, and also, according to Enskog theory, to the radial distribution function at contact distance [74]. If we assume, to simplify the problem, that these collisions are one-dimensional and perfectly elastic, then the kinetic energy transfer per collision between beads of mass $M_{1}$ and $M_{2}$ reads

$$
\begin{array}{r}
\Delta E_{K} \propto \frac{1}{\left(1+\frac{M_{1}}{M_{2}}\right)^{2}}\left[\frac{M_{1}}{M_{2}} k_{B} T_{1}-\frac{M_{1}}{M_{2}} k_{B} T_{2}+\right. \\
\left.+\sqrt{\frac{M_{1}}{M_{2}}}\left(1-\frac{M_{1}}{M_{2}}\right) k_{B} \sqrt{T_{1} T_{2}}\right],
\end{array}
$$

where $T_{1}$ and $T_{2}$ are the average temperatures of the contiguous layers containing the beads of mass $M_{1}$ and $M_{2}$, respectively. We refer the reader to the Supporting Information for the derivation of the above relation.

When a large mass $M_{2}$ collides against a small mass $M_{1}$, the resulting heat transfer per collision is practically negligible as $\Delta E_{K} \approx 0$ for $M_{2}>>M_{1}$. This would be the scenario observed in systems containing large NPs, with diameter $\sigma_{n}>>R_{g}$, impinging on polymer chain beads. Collisions between beads of identical mass generate a kinetic energy change per collision that only depends on the temperature gradient between the contiguous layers: $\Delta E_{K} \propto k_{B}\left(T_{1}-T_{2}\right)$. This small, but not negligible, temperature difference is, in this case, the sole driving force for the heat transfer. When the number of collisions per unit time and layer is relevant, then the total kinetic energy change and thus the heat transfer is relevant as well. In systems comprising large NPs, the value of the NP/NP radial distribution function at contact distance, $g_{n n}\left(\sigma_{n}\right)$, is basically zero (see for instance Fig. 3a), and therefore the corresponding NP/NP collisions are very rare and do not contribute to the transfer of heat. By contrast, the collisions between chain beads are much more frequent and determine the thermal conductivity of the PNC. We note that $\kappa$ is slightly lower than that of the corresponding pristine polymer, $\kappa_{0}$, in agreement with the above mentioned models describing the thermal conductivity of conventional composites loaded with micron-sized particles (shaded areas in Fig. 9). At decreasing NP size and mass, the value of the NP/NP radial distribution function at contact distance (Fig. 3d) and the NP/polymer interface area (Eq. 29) increase, suggesting that an important contribution to the total exchange of kinetic energy arises from collisions involving NPs. Consequently, smaller NPs, despite their non-conductive nature, actively contribute to the heat transfer through the polymer matrix and provoke an increase of the thermal conductivity up to $35 \%$ as compared to that of the pristine polymer melt. Our findings are in qualitative agreement with the experimental observations by Shima and Philip, who studied the effect of adding a very low amount of iron oxide $\left(\kappa=9.7 \mathrm{Wm}^{-1} \mathrm{~K}^{-1}\right)$ or silver $\left(\kappa=429 \mathrm{Wm}^{-1} \mathrm{~K}^{-1}\right)$ NPs (size 7 to $10 \mathrm{~nm}$ ) to hexadecane or kerosene [45]. Despite the clear difference in their thermal conductivity, the two sets of NPs have a substantially identical effect on the thermal conductivity of the nanofluids, which was shown to depend strongly on the NP volume fraction. However, the increase observed in our results, though interesting from the theoretical point of view, is still far from being practically relevant as compared to the thermal performance of most metals [75].

\section{CONCLUSIONS}

In summary, we investigated the intriguing effect of incorporating polydisperse NPs into a polymer melt of unentangled chains from single-particle to macroscopic transport properties. Across this wide spectrum of length scales, size dispersity displays a gradually decreasing influence that is relevant at the nanoscale and secondary at the macroscale. In an attempt to reproduce the typical features of a PNC, our NPs form attractive interactions with the polymer, their average size is of the order of the polymer radius of gyration, and their volume fraction, the same in all the systems studied, set to $5 \%$. Under these conditions, we found that polydispersity controls, along with the NP size, the mobility of the NPs 
in the melt and enhances their diffusivity as compared to that calculated for monodisperse systems. Our simulation results have been compared with existing theories on the diffusion of micron-sized particles that are here modified to incorporate the effect of size dispersity. We validated these theoretical models by calculating the diffusion coefficients of NPs over a range of particle's diameters and size dispersities. In particular, we detected a sound quantitative agreement that is especially good when the theories explicitly account for the non-continuous nature of the medium by introducing a nonhydrodynamic contribution, which is dominant for $\sigma_{n}<R_{g}$. The diffusion coefficients of the polymer chains are observed to fall into a single master curve when plotted against the interparticle distance, which depends on the NP average diameter and size dispersity. This result, previously observed experimentally [49], unveils an intriguing universal behavior that limits the dependence of the polymer chains' diffusivity on a single parameter and thus help us assess the complexity of the diffusion mechanism more efficiently, at least for the set of PNCs assessed in this study.

A similar universal behavior has been detected for the shear viscosity and thermal conductivity. The former does not display a particularly important dependence on size dispersity, but is found to be strongly related to the particle/polymer specific interface area, $s_{v}$, which increases with decreasing particle size and polydispersity. When plotted against $s_{v}$, the shear viscosity can be described by a single master curve showing a gradually increasing thickening effect that is especially visible in PNCs with very small NPs. We conclude that, more important than its geometric features, is the nature of interaction that the filler establishes with the neighboring polymer chains that provides a more complete insight into the viscosity of a PNC. We are currently working to consolidate these preliminary observations.

When investigating the thermal conductivity, we also noticed a weak dependence on the polydispersity of our NPs, which can basically be regarded as a completely insulating phase. As a general tendency, supported by several theoretical models describing the effect of micron-sized particles on the thermal properties of a polymer [37, 39, 40, 73], the thermal conductivity was found to be lower than that of the corresponding pristine polymer melt. Nevertheless, in systems where $\sigma_{n} \leq R_{g}$, despite the non-conductive nature of the NPs, the thermal conductivity of the PNC becomes larger than that of the pure polymer. We propose a simple theoretical description, based on the collisions between the beads in the system and determining a kinetic energy transfer between them, to explain this interesting result, where the NP mass plays a dominant role. These observations are in qualitative agreement with recent experiments showing that adding iron oxide or silver NPs does not determine any significant enhancement of the thermal conductivity of hexadecane or kerosenebased nanofluids at low NP volume fractions [45]. Also in this case, we could appreciate the existence of a universal behavior when plotting the thermal conductivity as a function of the inverse NP mass, $M_{n}$. Regardless of the NP size and poly- dispersity, our predictions collapse onto a single master curve that retrieves at $M_{n}>>M_{m}$ the thermal conductivity of a PNC incorporating micron-sized fillers.

\section{ACKNOWLEDGMENTS}

JJBM acknowledges a postgraduate grant from the University of Manchester (AA02879). The authors would like to acknowledge the assistance given by IT Services and the use of the Computational Shared Facility at The University of Manchester.

\section{SUPPLEMENTAL MATERIAL}

Supplemental material is available. This includes additional details on the (i) probability density functions, (ii) equilibration procedure, (iii) RNEMD, (iv) the $O L$ model for $\sigma_{c} / \sigma_{m}<1$, (v) diffusivities as obtained from the $Y S$ and $O L$ models, and (vi) elastic collisions between beads.

* Electronic mail: alessandro.patti@manchester.ac.uk

[1] Paul, D. R.; Robeson, L. M. Polymer Nanotechnology: Nanocomposites. Polymer 2008, 49, 3187-3204. DOI: 10.1016/j.polymer.2008.04.017.

[2] Njuguna, J.; Pielichowski, K. Polymer Nanocomposites for Aerospace Applications: Properties. Adv. Eng. Mater. 2003, 5, 769-778. DOI: 10.1002/adem.200310101.

[3] Hule, R. A.; Pochan, D. J. Polymer Nanocomposites for Biomedical Applications. MRS Bull. 2007, 32, 354-358. DOI: 10.1557/mrs2007.235.

[4] Crosby, A. J.; Lee, J.-Y. Polymer Nanocomposites: the "Nano" Effect on Mechanical Properties. Polym. Rev. (Philadelphia, PA, U. S.) 2007, 47, 217-229. DOI: 10.1080/15583720701271278.

[5] De Volder, M. F. L.; Tawfick, S. H.; Baughman, R. H.; Hart, A. J. Carbon Nanotubes: Present and Future Commercial Applications. Science (Washington, DC, U.S.) 2013, 339, 535-539. DOI: $10.1126 /$ science. 1222453.

[6] Mittal, V. Polymer nanocomposite coatings, 1st Ed., CRC Press, 2013.

[7] Qi, X.; Tan, C.; Wei, J.; Zhang, H. Synthesis of graphene-conjugated polymer nanocomposites for electronic device applications. Nanoscale 2013, 5, 1440-1451. DOI: 10.1039/C2NR33145D.

[8] Rhim, J.-W.; Park, H.-M.; Ha, C.-S. Bio-nanocomposites for food packaging applications. Prog. Polym. Sci. 2013, 38, 16291652. DOI: 10.1016/j.progpolymsci.2013.05.008.

[9] Huang, X.; Jiang, P. Core-Shell Structured High- $k$ Polymer Nanocomposites for Energy Storage and Dielectric Applications. Adv. Mater. 2015, 27, 546-554. DOI: 10.1002/adma.201401310.

[10] Crowley, C.; Birchall, M.; Seifalian, A. M. Trachea transplantation: from laboratory to patient. J. Tissue Eng. Regener. Med. 2015, 9, 357-367. DOI: 10.1002/term.1847.

[11] X. J. Loh, Polymers for personal care and cosmetics, 1st Ed., The Royal Society of Chemistry, 2016. 
[12] Tuteja, A.; MacKay, M. E. Breakdown of the Continuum Stokes-Einstein relation for Nanoparticle Diffusion. Nano Lett. 2007, 7, 1276-1281. DOI: 10.1021/n1070192x.

[13] Grabowsky, C. A.; Mukhopadhyay, A. Size Effect of Nanoparticle Diffusion in a Polymer Melt. Macromolecules 2014, 47, 7238-7242. DOI: 10.1021/ma501670u.

[14] Araque, J. C.; Yadav, S. K.; Shadeck, M.; Maroncelli, M.; Margulis, C. J. How is Diffusion of Neutral and Charged Tracers Related to the Structure and Dynamics of a Room-Temperature Ionic Liquid? Large Deviations from Stokes-Einstein Behavior Explained. J. Phys. Chem. B 2015, 119, 7015-7029. DOI: 10.1021/acs.jpcb.5b01093.

[15] Ould-Kaddour, F.; Levesque, D. Molecular Dynamics investigation of tracer diffusion in a simple liquid: Test of the Stokes-Einstein law. Phys. Rev. E 2000, 63, 011205. DOI: 10.1103/PhysRevE.63.011205.

[16] Sharma, M.; Yashonath, S. Breakdown of the Stokes-Einstein Relationship: Role of Interactions in the Size Dependence of Self-Diffusivity. J. Phys. Chem. B 2006, 110, 17207-17211. DOI: 10.1021/jp064364a.

[17] Liu, J.; Cao, D.; Zhang, L. Molecular Dynamics Study on Nanoparticle Diffusion in Polymer Melts: A Test of the StokesEinstein Law. J. Phys. Chem. C 2008, 112, 6653-6661. DOI: 10.1021/jp800474t.

[18] Ganesan, V.; Pryamitsyn, V.; Surve, M.; Narayanan, M. Noncontinuum effects in nanoparticle dynamics in polymers. $J$. Chem. Phys. 2006, 124, 221102. DOI: 10.1063/1.2209241.

[19] Brochard-Wyart, F.; de Gennes, P. G. Viscosity at small scales in polymer melts. Eur. Phys. J. E 2000, 1, 93-97. DOI: 10.1007/s101890050011.

[20] Yamamoto, U.; Schweizer, S. Theory of nanoparticle diffusion in unentangled and entangled polymer melts. J. Chem. Phys. 2011, 135, 224902. DOI: 10.1063/1.3664863.

[21] Einstein, A. Eine neue Bestimmung der Moleküldimensionen. Ann. Phys. 1906, 324, 298-306. DOI: 10.1002/andp.19063240204.

[22] Einstein, A. Berichtigung zu meiner Arbeit: „Eine neue Bestimmung der Molekül-dimensionen". Ann. Phys. 1911, 339, 591-592. DOI: 10.1002/andp.19113390313.

[23] Roscoe, R. The viscosity of suspensions of rigid spheres. Br. J. Appl. Phys. 1952, 3, 267-269. DOI: 10.1088/05083443/3/8/306.

[24] Thomas, D. G. Transport characteristics of suspension: VIII. A note on the viscosity of newtonian suspensions of uniform spherical particles. J. Colloid Sci. 1965, 20, 267-277. DOI: 10.1016/0095-8522(65)90016-4.

[25] Batchelor, G. K. The effect of Brownian motion on the bulk stress in a suspension of spherical particles. J. Fluid Mech. 1977, 83, 97-117. DOI: 10.1017/S0022112077001062.

[26] Kholodenko, A. L.; Douglas, J. F. Generalized Stokes-Einstein equation for spherical particle suspensions. Phys. Rev. E 1995, 51, 1081-1090. DOI: 10.1103/PhysRevE.51.1081.

[27] Toda, K.; Furuse, H. Extension of Einstein's Viscosity Equation to that for Concentrated Dispersions of Solutes and Particles. $J$. Biosci. Bioeng. 2006, 102, 524-528. DOI: 10.1263/jbb.102.524.

[28] Mackay, M. E.; Dao, T. T.; Tuteja, A.; Ho, D. L.; Van Horn, B.; Kim, H.-C.; Hawker, C. J. Nanoscale effects leading to nonEinstein-like decrease in viscosity. Nat. Mater. 2003, 2, 762766. DOI: 10.1038/nmat999.

[29] Kalathi, J. T.; Grest, G. S.; Kumar, S. K. Universal Viscosity Behavior of Polymer Nanocomposites. Phys. Rev. Lett. 2012, 109, 198301. DOI: 10.1103/PhysRevLett.109.198301.

[30] Patti, A. Molecular Dynamics of Spherical Nanoparticles in Dense Polymer Melts. J. Phys. Chem. B 2014, 118, 3731-3742.
DOI: $10.1021 / \mathrm{jp} 412440 \mathrm{~g}$.

[31] Smith, G. D.; Bedrov, D.; Li, L.; Byutner, O. A molecular dynamics simulation study of the viscoelastic properties of polymer nanocomposites. J. Chem. Phys. 2002 117, 9478-9489. DOI: $10.1063 / 1.1516589$.

[32] Schmidt, R. G.; Gordon, G. V.; Dreiss, C. A.; Cosgrove, T.; Krukonis, V. J.; Williams, k.; Wetmore, P. M. A Critical Size Ratio for Viscosity Reduction in Poly(dimethylsiloxane)Polysilicate Nanocomposites. Macromolecules 2010, 43, 10143-10151. DOI: 10.1021/ma1004919.

[33] Idumah, C. I.; Hassan, A. Recently emerging trends in thermal conductivity of polymer nanocomposites. Rev. Chem. Eng. 2016; 32, 413-457. DOI: 10.1515/revce-2016-0004.

[34] Colonna, S.; Monticelli, O.; Gomez, J.; Novarad, C.; Saracco, G.; Fina, G. Effect of morphology and defectiveness of graphene-related materials on the electrical and thermal conductivity of their polymer nanocomposites. Polymer 2016, 102, 292-300. DOI: 10.1016/j.polymer.2016.09.032.

[35] S. Torquato, Random heterogeneous materials, 1st Ed., New York, Springer-Verlag, 2001.

[36] Chen, H.; Ginzburg, V. V.; Yang, J.; Yang, Y.; Liu, W.; Huang, Y.; Du, L.; Chen, B. Thermal conductivity of polymer-based composites: Fundamentals and applications. Prog. Polym. Sci. 2016, 59, 41-85. DOI: 10.1016/j.progpolymsci.2016.03.001.

[37] Smith, D. S.; Alzina, A.; Bourret, J.; Nait-Ali, B; Pennec, F.; Tessier-Doyen, N.; Otsu, K.; Matsubara, H.; Elser, P.; Gonzenbach, U. T. Thermal conductivity of porous materials. J. Mater. Res. 2013, 28, 2260-2272. DOI: 10.1557/jmr.2013.179.

[38] Hasselman, D. P. H.; Johnson, L. F. Effective Thermal Conductivity of Composites with Interfacial Thermal Barrier Resistance. J. Comp. Mat. 1987, 21, 508-515. DOI: 10.1177/002199838702100602.

[39] Benvensite, Y. Effective thermal conductivity of composites with a thermal contact resistance between the constituents: Nondilute case. J. App. Phys. 1987, 61, 2840-2843. DOI: 10.1063/1.337877.

[40] Nan, C.-W.; Birringer, R.; Clarke, D. R.; Gleiter, H. Effective thermal conductivity of particulate composites with interfacial thermal resistance. J. App. Phys. 1997, 81, 6692-6699. DOI: 10.1063/1.365209.

[41] Ordóñez-Miranda, J. ; Alvarado-Gil, J. J. Medina-Ezquivel, R.; Generalized Bruggeman Formula for the Effective Thermal Conductivity of Particulate Composites with an Interface Layer. Int. J. Thermophys. 2010, 31, 975-986. DOI: 10.1007/s10765010-0756-2.

[42] Minnich, A.; Chen, G. Modified effective medium formulation for the thermal conductivity of nanocomposites. Appl. Phys. Lett. 2007, 91, 073105. DOI: 10.1063/1.2771040.

[43] Bruggeman, D. A. G. Berechnung verschiedener physikalischer Konstanten von heterogenen Substanzen. Ann. Phys. 1935, 24, 636-664. DOI: 10.1002/andp.19354160705.

[44] Ordóñez-Miranda, J.; Alvarado-Gil, J. J. Thermal conductivity of nanocomposites with high volume fractions of particles. Compos. Sci. Technol. 2012, 72, 853-857. DOI: 10.1016/j.compscitech.2012.02.016.

[45] Shima, P. D.; Philip, J. Role of Thermal Conductivity of Dispersed Nanoparticles on Heat Transfer Properties of Nanofluid. Ind. Eng. Chem. Res. 2014, 50, 980-988. DOI: 10.1021/ie403086g.

[46] Sciortino, F.; Tartaglia, P. Glassy colloidal systems. Adv. Phys. 2005, 54, 471-524. DOI: 10.1080/00018730500414570.

[47] Berthier, L.; Biroli, G.; Bouchaud, J.-P.; Cipelletti, L.; Van Saarloos, W. Dynamical heterogeneities in glasses, colloids, and granular media, 1st Ed., Oxford Science Publications, 
2011.

[48] Fullstone, G.; Wood, J.; Holcombe, M.; Battaglia, G. Modelling the Transport of Nanoparticles under Blood Flow using an Agent-based Approach. Sci. Rep. 2015, 5, 10649. DOI: 10.1038/srep10649.

[49] Gam, S.; Meth, J. S.; Zane, S. G.; Chi, C.; Wood, B. A.; Winey, K. I.; Clarke N.; Composto, R. J.; Polymer diffusion in a polymer nanocomposite: effect of nanoparticle size and polydispersity. Soft Matter 2012, 8, 6512-6520. DOI: 10.1039/C2SM25269D.

[50] Cadel, R.D. Particle Size. Theory and industrial applications. Reinhold Pub. Corp. 1965.

[51] Lin, C.-C.; Ohno, K.; Clarke, N.; Winey, K. I.; Composto, R. J. Macromolecular Diffusion through a Polymer Matrix with Polymer-Grafted Chained Nanoparticles. Macromolecules 2014, 47, 5357-5364. DOI: 10.1021/ma501113c.

[52] Although IUPAC recommends to use the mass-molar dispersity $\bigoplus_{M}$ (in our case, the diameter dispersity $\bigoplus_{\sigma_{n}}$ ) instead of the polydispersity index, $\Pi_{D}[53]$, we choose to use the latter throughout this article, as defined in Eq. (5), to avoid confusion with the diffusion of particles, $D_{n}$. These parameters are related through $\bigoplus_{\sigma_{n}}=1+\Pi_{Ð}$, and $\bigoplus_{M_{n}}=\left(1+\Pi_{Ð}\right)^{9}$.

[53] IUPAC, Polymer Division, Subcommittee on Polymer Terminology. Dispersity in polymer science (IUPAC Recommendations 2009). Pure Appl. Chem. 2009, 81, 351-353. DOI: 10.1002/pi.2748.

[54] Kremer, K.; Grest, G. S. Dynamics of entangled linear polymer melts: A molecular-dynamics simulation. J. Chem. Phys. 1990, 92, 5057-5086. DOI: 10.1063/1.458541.

[55] Smith, J. S.; Bedrov, D.; Smith, G. D. A molecular dynamics simulation study of nanoparticle interactions in a model polymer-nanoparticle composite. Compos. Sci. Technol. 2003, 63, 1599-1605. DOI: 10.1016/S0266-3538(03)00061-7.

[56] Humphrey, W.; Dalke, A.; Schulten, K. VMD: Visual Molecular Dynamics. J. Molec. Graphics 1996, 14, 33-38. DOI: 10.1016/0263-7855(96)00018-5.

[57] Mathematica, version 10.3; software for technical computation; Wolfram Research: Champaign, IL 2015.

[58] LAMMPS - Large-scale Atomic/Molecular Massively Parallel Simulator, versions Feb 14 and May 15; Plimpton, S. J. Comput. Phys. 1995, 117, 1-19. DOI: 10.1006/jcph.1995.1039.

[59] Rubinstein, M.; Colby R. H. Polymer Physics, Oxford University Press, 2003.

[60] Doi, M.; Edwards, S. F. The theory of polymer dynamics, Oxford University Press, 1986.

[61] Müller-Plathe, F. Reversing the perturbation in nonequilibrium molecular dynamics: An easy way to calculate the shear viscosity of fluid. Phys. Rev. E 1999, 59, 4894-4898. DOI: 10.1103/PhysRevE.59.4894.

[62] Müller-Plathe, F. A simple nonequilibrium molecular dynamics method for calculating the thermal conductivity. J. Chem. Phys. 1997, 106, 6082-6085. DOI: 10.1063/1.473271.

[63] Müller-Plathe, F.; Bordat, P.; Novel Methods in Soft Matter Simulations, in Lect. Notes Phys. 2004, 640, 310-326.

[64] Chen, X.; Carbone, P.; Cavalcanti, W. L.; Milano, G.; MüllerPlathe, F.; Viscosity and Structural Alteration of a CoarseGrained Model of Polystyrene under Steady Shear Flow Studied by Reverse Nonequilibrium Molecular Dynamics. Macromolecules 2007, 40, 8087-8095. DOI: 10.1021/ma0707178.

[65] Dünweg, B.; Kremer, K. Molecular dynamics simulation of a polymer chain in solution. J. Chem. Phys. 1993, 99, 6983-6997. DOI: $10.1063 / 1.465445$.

[66] Yeh, I.-C.; Hummer, G. System-size dependence of diffusion coefficients and viscosities from Molecular Dynamics simulations with periodic boundary conditions. J. Phys. Chem. B 2004, 108, 15873-15879. DOI: 10.1021/jp0477147.

[67] Batchelor, G. K. Brownian diffusion of particles with hydrodynamic interaction. J. Fluid Mech. 1976, 74, 1-29. DOI: 10.1017/S0022112076001663.

[68] Beenakker, C. W. J.; Mazur, P. Diffusion of spheres in a concentrated suspension II. Physica A 1984, 126, 349-370. DOI: 10.1016/0378-4371(84)90206-1.

[69] Ando, T.; Chow, E.; Skolnick, J. Dynamic simulation of concentrated macromolecular solutions with screened longrange hydrodynamic interactions: Algorithm and limitations. J. Chem. Phys. 2013, 139, 121922. DOI: 10.1063/1.4817660.

[70] Chantrenne, P.; Barrat, J.-L. Finite size effects in determination of thermal conductivities: Comparing Molecular Dynamics results with simple models. J. Heat Transf. 2004, 126, 577-585. DOI: 10.1115/1.1777582.

[71] Li, Y.; Kröger, M.; Liu, W. K. Dynamic Structure of Unentangled Polymer Chains in the Vicinity of Non-attractive Nanoparticles. Soft Matter 2014, 10, 1723-1737. DOI: 10.1039/C3SM51564H.

[72] Hamilton, R.; Crosser, O. Thermal conductivity of heterogeneous two-component systems. Ind. Eng. Chem. Fund. 1962, 3, 187-191. DOI: 10.1021/1160003a005.

[73] Landauer, R. The Electrical Resistance of Binary Metallic Mixtures. J. App. Phys. 1952, 23, 779-784. DOI: 10.1063/1.1702301.

[74] Hansen J.-P.; McDonald, I. R. Theory of Simple Liquids, 3rd Ed., Academic Press, 2006.

[75] Lide, D. R. (ed) CRC Handbook of Chemistry and Physics, 84th Ed., CRC Press, 2003. 